\title{
Review
}

Jialin Ma and Mengtao Sun*

\section{Nonlinear optical microscopies (NOMs) and plasmon-enhanced NOMs for biology and 2D materials}

https://doi.org/10.1515/nanoph-2020-0082

Received February 3, 2020; revised March 9, 2020; accepted March 17, 2020

\begin{abstract}
In this review, we focus on the summary of nonlinear optical microscopies (NOMs), which are stimulated Raman scattering (SRS), coherent anti-Stokes Raman scattering (CARS), second harmonic generation (SHG), and two-photon excited fluorescence (TPEF). The introduction is divided into two parts: the principle of SRS, CARS, TPEF, and SHG and their application to biology and two-dimensional materials. We also introduce the connections and differences between them. We also discuss the principle of plasmon-enhanced NOM and its application in the above two aspects. This paper not only summarizes the research progress in the frontier but also deepens the readers' understanding of the physical principles of these NOMs.
\end{abstract}

Keywords: nonlinear optical microscopies; two-photon excited fluorescence; stimulated Raman scatting; second harmonic generation; coherent anti-Stokes Raman scattering.

\section{Introduction}

With the development of microscopy, optical microscopy has become a significant technology in biology and

\footnotetext{
*Corresponding author: Mengtao Sun, School of Mathematics and Physics, Center for Green Innovation, Beijing Advanced Innovation Center for Materials Genome Engineering, Beijing Key Laboratory for Magneto-Photoelectrical Composite and Interface Science, University of Science and Technology Beijing, No. 30 Xueyuan Road, Beijing 100083, P.R. China, e-mail: mengtaosun@ustb.edu.cn. https://orcid.org/0000-0002-8153-2679

Jialin Ma: School of Mathematics and Physics, Center for Green Innovation, Beijing Advanced Innovation Center for Materials Genome Engineering, Beijing Key Laboratory for MagnetoPhotoelectrical Composite and Interface Science, University of Science and Technology Beijing, Beijing 100083, P.R. China
}

materials science in recent years. A lot of problems can only be resolved by proper microscopy [1]. This fact is most evident in biology. By combining the characteristics of spectrum and microscopy, dynamic processes, such as nucleic acids, proteins, lipids, and so on, can be observed with lossless and direct methods [2]. New tools in microbiology often promote the development of biological sciences, and nonlinear optical microscopes (NOMs) play an important role. In the past decade, nonlinear optical technology has been widely used in the detection of biomolecules at micron and nanometer levels $[3,4]$ as well as in other fields, such as medicine and pharmacy [5-7]. In contrast to optical methods, radiation is commonly used in imaging methods, such as X-ray, magnetic resonance imaging, and other nuclear imaging techniques [8], but these technologies are expensive and emit radiation. At this point, optical imaging technology shows its own advantages. Optical methods can provide noninvasive, immediate, exact, and economical molecular information of human tissues during disease detection, diagnosis, and cell development. In addition, nonlinear optical methods have the advantages of deep penetration, great sensitivity, and ultrahigh resolution [9-11]. The nonlinear signal is excited by a laser using a picosecond or femtosecond near-infrared pulse using an NOM. The advantages of NOMs are obvious in scientific research.

Maker and Terhune first reported on coherent antiStokes Raman scattering (CARS) in 1965 [12], which is a nonlinear optical process. Later, Begley named it as CARS in 1974. From then on, as a spectral analysis method, CARS spectroscopy has been used extensively in gasphase chemical analysis and condensed matter, making it the most widely used nonlinear Raman spectral analysis technique. CARS is a third-order nonlinear optical method. CARS microscopy, as a new nonlinear optical technique, puts vibration spectroscopy in combination with microscopy that allows for high-sensitivity studies of samples that do not require marking. CARS is a perfect tool in studying different samples. Fluorescence microscopy is already a powerful and widespread technique for the study 
of cell biology, but it has two disadvantages, such as the photobleaching of fluorescent probes and the effect of fluorescent probes on cell functions. These difficulties can be avoided by multiphoton microscopy based on CARS.

Stimulated Raman scattering (SRS) is a third-order nonlinear optical method and is a type of Raman scattering. In SRS, due to the optical amplification of the vibration excitation rate, the imaging speed of an SRS microscope increases by an order of magnitude compared to that of a spontaneous Raman microscope, which is a great breakthrough. SRS can be used as a contrast technique, and its use in microscopes was first reported in 2007 [13]. Using multiple detection of a photodiode array in a femtosecond laser amplification system, SRS achieves unlabeled vibration specificity, extraordinary imaging speed, and outstanding detection sensitivity. As published in other papers, living cells do not require external markers but simple marking of different chemical components for imaging [14, 15]. Marking various vibrational bands can also be applied widespread in chemical imaging in the biomedical field.

Here, we also introduce second-order nonlinear optical processes, which are second harmonic generation (SHG), sum frequency generation (SFG), and two-photon excited fluorescence (TPEF). SHG was first discovered by Franken in 1961. It is also widely applied in detecting biological molecules, testing drugs, medical diagnosis, and other fields [16]. It plays an important role in the detection of tissues and living cells. Usually, SHG is used to measure and image the contractile integrity of muscles. There are similarities and differences between TPEF and SHG; the former is a third-order nonlinear optical method, and the penetration depth to detect living cells and tissues can be limited to $1 \mathrm{~mm}$ [17]. This technique has high sensitivity and resolution. TPEF combined with a microscope can be used to diagnose and detect the metabolic activity, morphological changes, and protein expression of cancer cells [18]. In this review, we will cover the four NOMs and the plasmon-enhanced NOMs, although not completely.

\section{Principle of NOMs}

In biology and medicine, light microscopy is limited by phototoxicity. Compared to the linear process, the nonlinear process can provide instantaneous, particularly high pulse excitation light with lower average energy on the living sample. To accurately learn NOM, we introduce the relationship between linear optical polarization and electric field intensity, and it can be described by the following equation:

$$
P(t)=\chi^{(1)} E(t)
$$

where $P(t)$ is the polarization and $\chi^{(1)}$ is the linear susceptibility. For a better understanding of NOMs, according to the linear light formula mentioned above, the author correctly represented the relationship between the electric field intensity and the polarization of the NOMs, that is [19],

$$
P(t)=\varepsilon_{0}\left[\chi^{(1)} E(t)+\chi^{(2)} E(t)+\chi^{(3)} E(t)+\cdots\right]
$$

where $\varepsilon_{0}$ is the free space permittivity and $\chi^{(2)}$ and $\chi^{(3)}$ are the second- and third-order susceptibility, respectively. Therefore, second-order nonlinear optics are described using the following equation:

$$
P\left(\omega_{\mathrm{pr}}+\omega_{\mathrm{p}}\right)=2 \varepsilon_{0} \chi^{(2)} E_{1} E_{2}
$$

\subsection{Principle of CARS and SRS}

CARS is a coherent technique, CARS microscopy has some advantages of obtaining stronger signals than spontaneous Raman scattering microscopy, and most importantly, these signals are detected with high energy compared to the excitation wavelength. Thus, the fluorescence background of the samples will not hinder the detection of the signals. The results show that, because of these properties, CARS microscopy has developed an attractive technique for microstudying various samples. In 1965, CARS was first reported at Ford Motor Company by Maker and Terhune; it was a nonlinear optical process. Begley et al. later named CARS in 1974. From then on, CARS spectroscopy had been broadly applied as a spectral exploration implement in chemical analysis, physics, biology, and materials science [12, 20-22] and had become the most generally used nonlinear Raman technique. Figure 1 demonstrates its energy-level diagram. The CARS spectrum uses multiple photons to motivate Raman active molecular vibrations following in the coherent Raman signal. In this system, a pump light with frequency $\omega_{\mathrm{p}}$, a probe laser with frequency $\omega_{\mathrm{pr}}$, and a Stokes light with frequency $\omega_{\mathrm{s}}$ are tightly intensive on the sample to produce a CARS signal with frequency $\omega_{\mathrm{pr}}+\omega_{\mathrm{p}}-\omega_{\mathrm{s}}$. When $\omega_{\mathrm{pr}}=\omega_{\mathrm{p}}$, the anti-Stokes frequency can be expressed as $2 \omega_{\mathrm{p}}-\omega_{\mathrm{s}}$; when the frequency difference $\omega_{\mathrm{p}}-\omega_{\mathrm{s}}$ is modified to the Raman-activated molecular pulsation, an intensely resonant CARS signal is produced. This means that it can 
CARS

energy diagram

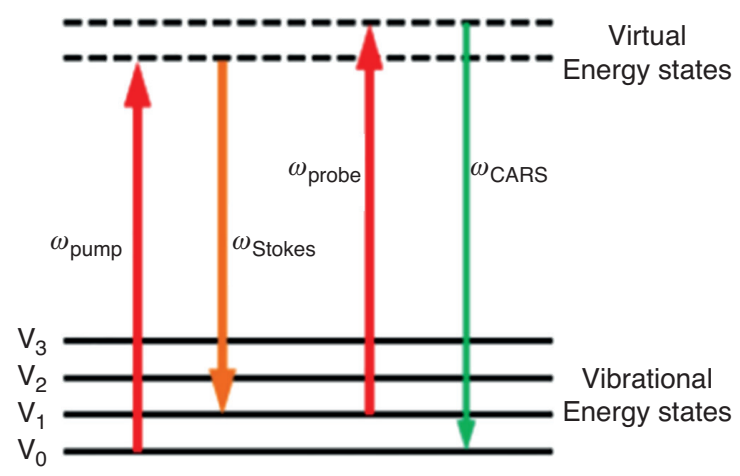

Figure 1: Energy diagrams of CARS [23].

provides biochemical selectivity for CARS microscopy. The light path diagram of the CARS device and microscope is shown in Figure 2.

Based on CARS microscopy, an electro-optical or acoustic-optical modulator is added to the Stokes beam, and a phase-locked amplifier is added to the photomultiplier tube to generate the SRS signal. SRS is a third-order nonlinear optical method that is a type of Raman scattering, and it uses femtosecond or picosecond lasers to image vibrational fingerprints of living cells and tissues directly. SRS provides high-speed, high-resolution, and high-sensitivity, free labeling, real-time, and 3D properties to image the distribution of specific molecules [24] and tumors cells [25] and can detect intracellular extracts
[26-29]. In SRS, pumped photons are converted to Stokes photons when molecules are motivated from the ground vibrational state to the first vibrational excited state [30] as shown in Figure 3.

The relationship between electric field strength and polarization of SRS and CARS can be expressed as

$$
\begin{gathered}
P\left(2 \omega_{\mathrm{pr}}-\omega_{\mathrm{p}}\right)=3 \varepsilon_{0} \chi^{(3)} E_{1}^{2} E_{2}^{*} \\
P\left(\omega_{\mathrm{pr}}+\omega_{\mathrm{p}}-\omega_{s}\right)=6 \varepsilon_{0} \chi^{(3)} E_{1} E_{2} E_{3}^{*}
\end{gathered}
$$

\subsection{Principle of SHG and TPEF}

SHG, SFG, and TPEF are all second-order nonlinear optical processes, and SHG converts two photons of one kind of wavelength into one photon at half the wavelength of the original photon. In other words, as second-order nonlinear optical techniques with threewave mixing, when $\omega_{\mathrm{pr}}=\omega_{\mathrm{p}}$, it is defined as SHG, and when $\omega_{\mathrm{pr}} \neq \omega_{\mathrm{p}}$, it is defined as SFG. The energy levels of SHG and SFG are shown in Figure 4. Franken was the first one to discovered SHG in 1961. Because it uses the same laser scanning microscope and laser light source plus an appropriate narrowband filter, it can be combined with other nonlinear devices. It is widely used in biological molecular detection, medical diagnosis, and drug testing. Due to its good physical properties, it is very sensitive not only to the structures of collagen fibers

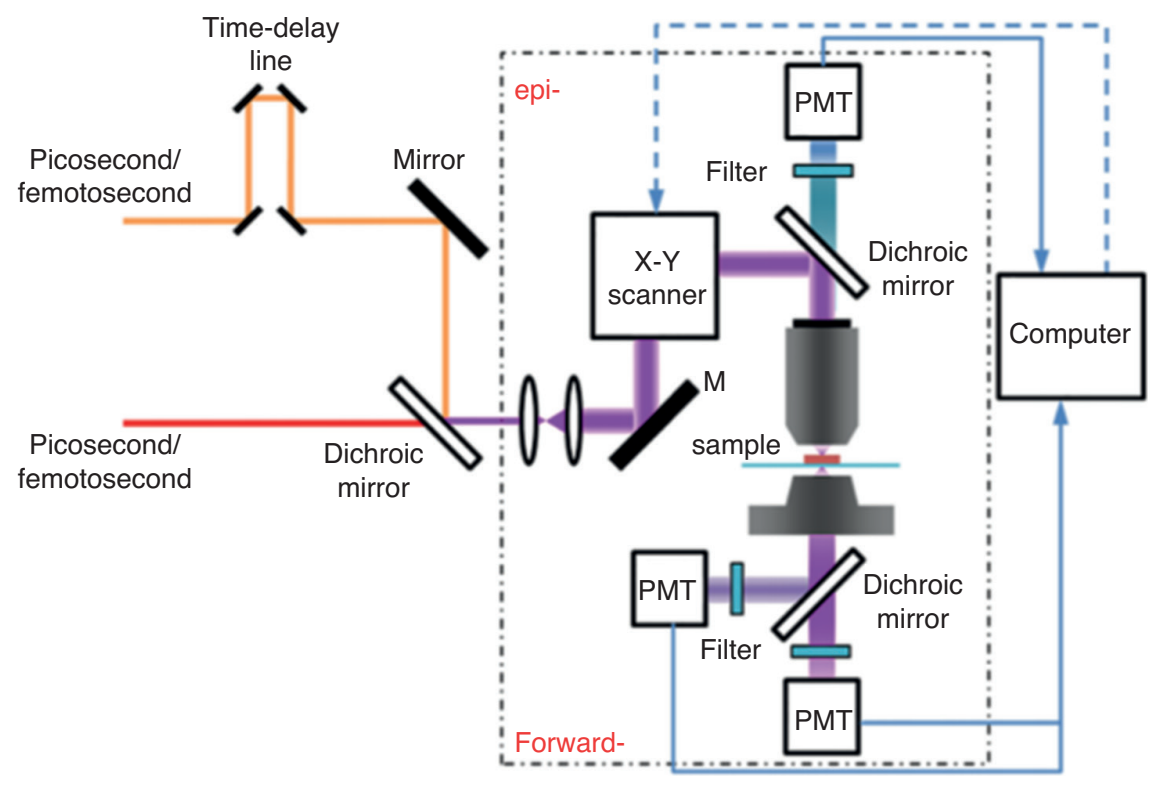

Figure 2: Optical path diagram of a CARS microscope [4]. 


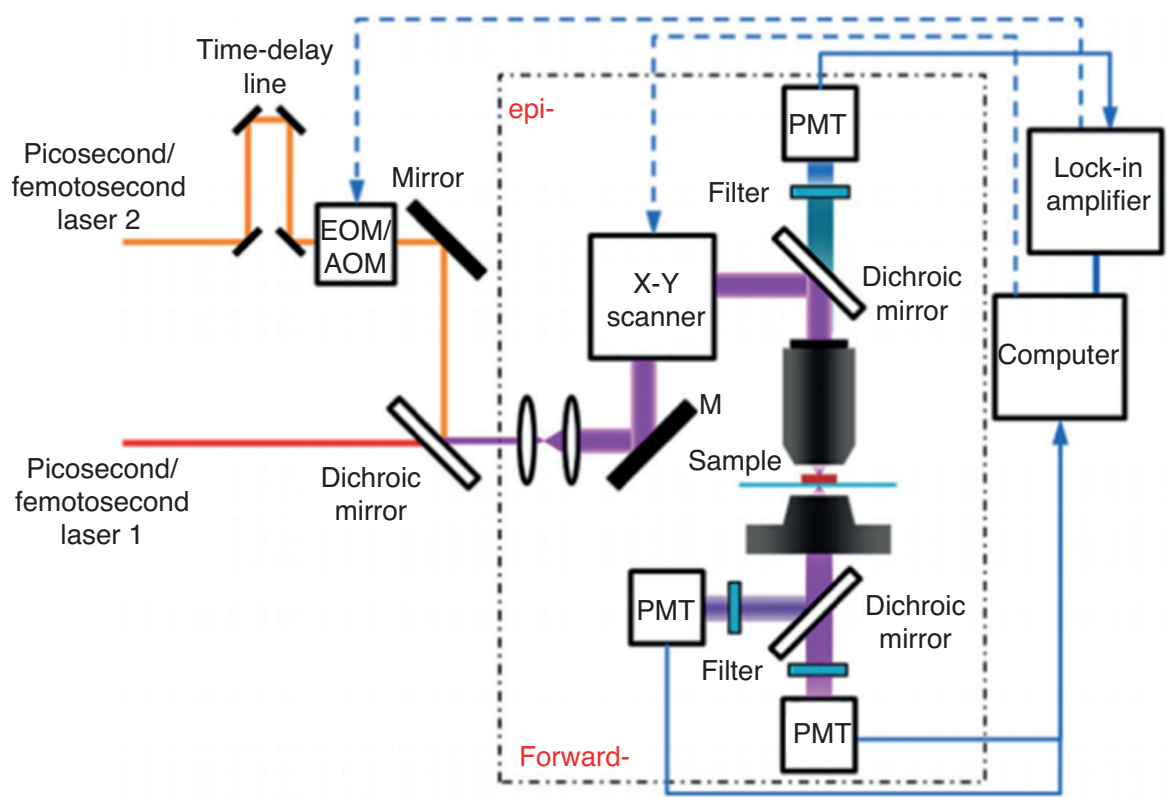

Figure 3: Optical path diagram of an SRS microscope [4].

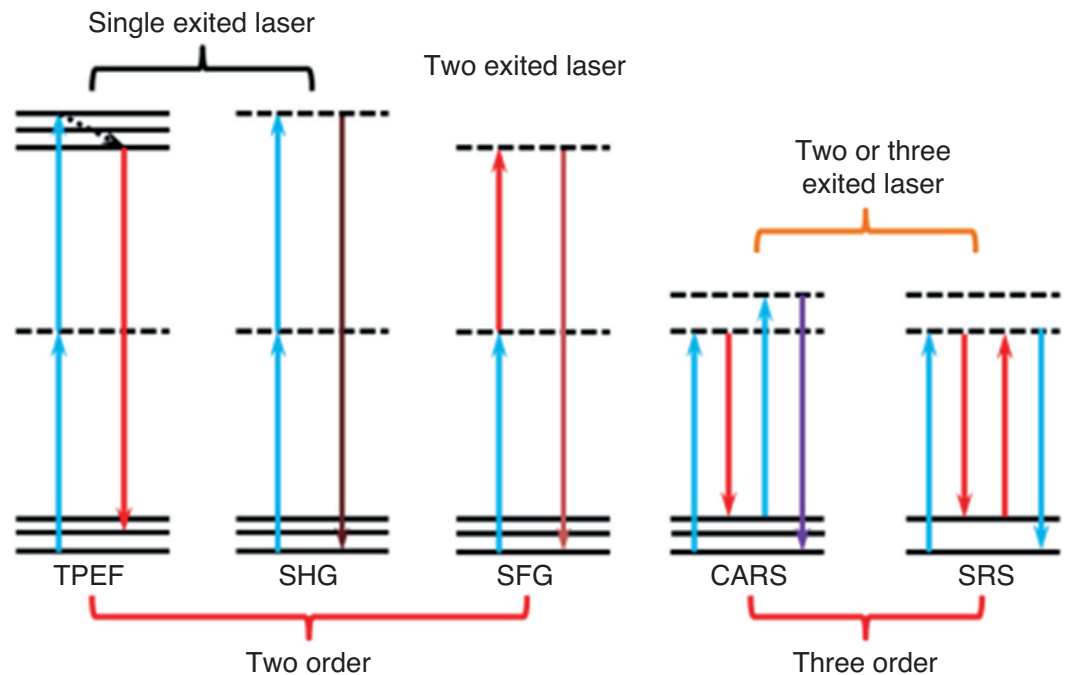

Figure 4: Energy-level diagram for nonlinear optical process of TPEF, SHG, SFG, CARS, and SRS processes [4].

but also to the changes that occur in diseases such as cancer, fibrosis, and connective tissue diseases [31-39]. It is noted that SHG only corresponds to materials that have no inversion symmetry.

TPEF microscopy is one of the traditional fluorescence imaging methods. Focusing a picosecond or femtosecond beam on a sample with a scanning microscope generates a fluorescence signal. Combining TPEF microscopy with fluorescent materials provides rapid techniques for diagnosing and monitoring various diseases using encoded fluorescent proteins, exogenous dyes, and nanomaterials [40]. The working mechanism of TPEF and SHG microscopes is shown in Figure 5. TPEF and SHG can be combined to achieve better imaging results. TPEF comes from specific molecules, and it can be used to study how molecules are arranged in fibers. SHG is a property inherent in specific structures, and it can be used to study the dynamic process of molecules, so combining the two can provide more comprehensive information. 


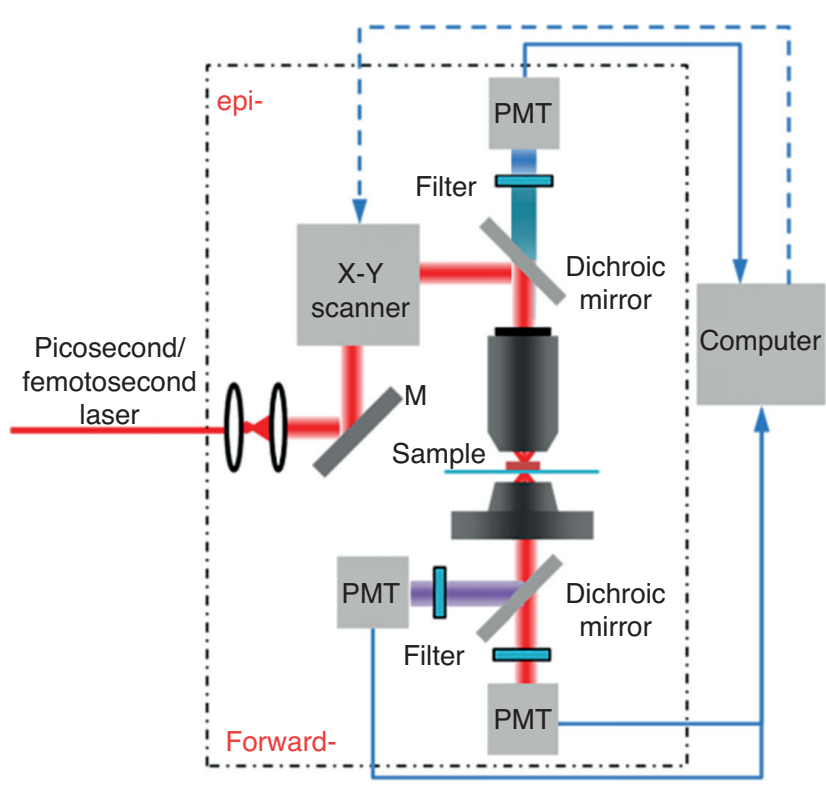

Figure 5: Optical path diagram of an SHG or TPEF microscope [4].

\section{Applications of NOMs in biology}

\subsection{Applications of CARS}

In the application of CARS microscopy mentioned above, it can be used to monitor material changes in cancer cells. Lipid synthesis plays a crucial role in cell proliferation, cell division, and cell cycle growth [41], so it is necessary for cancer cells to study changes in the composition and content of lipids. The global incidence of breast cancer has been on the rise since the late 1970s, it has been the most common malignancy, and it is also one of the main factors of cancer deaths among American women [42]. In addition, prostate cancer is the first common malignant tumor in American males [43]. Potcoava et al. used Raman and CARS microscopy to investigate the changes in the lipid composition and content in breast and prostate cancer cells that are cured by hormones [44]. The signal strength of CARS represents the amount of lipid content. Quantitative analysis of lipids in cells is often performed by disruptive chromatography [45], which cannot offer 3D information on lipid distribution, and it cannot be monitored in living cells and in vivo. Raman spectroscopy can also tell whether cells are healthy or cancerous [46, 47]. They studied whether the lipid content and composition of breast (T47D and MDA-MB-231) cancer cells are affected by the synthesis of the female hormone medroxyprogesterone acetate (MPA), and prostate (LNCaP and PC3) cancer cells are affected by the synthesis of androgen R1881. They compared two groups: (1) T47D and LNCaP treated with hormones and (2) those not cured by hormone. The Raman spectral diagram is shown in Figure 6.

Pictures $\mathrm{a}$ and $\mathrm{b}$ were obtained from breast cancer cell lines individually in the low- and high-wavenumber regions of the spectrum, respectively. Panels $\mathrm{c}$ and $\mathrm{d}$ were the low- and high-wavenumber spectra of prostate cancer cell lines, respectively. The difference spectrum (mean subtraction) is also shown at the bottom of each graph. The most significant changes between the spectral treated cells and controls occurred in T47D and LNCaP cell samples peaked at $1439 \mathrm{~cm}^{-1}$ (b and c) and high-wavenumber regions (b and d). MDA-MB-231 did not display a significant difference between the two groups, suggesting a smaller response to hormone therapy. Because T47D and LNCaP were more responsive to hormone therapy, they recorded the CARS and TPEF images for treated and untreated hormone-sensitive cancer cells, respectively (Figures 7 and 8). Consequently, the Raman-based approach is a significant part in detecting what effects of hormone and cholesterol levels on cancer development are probable. Noninvasive Raman technology is similarly expected to give help in the clinical monitoring and exploration of breast and prostate cancers.

CARS microscopy also is used for generating molecular-specific distinction grounded on vibration spectra of sample molecules as a new tool. Because of the production of vibration contrast agent, samples do not need to be stained. As a kind of coherent technique, it shows the properties of obtaining more obvious signals than spontaneous Raman scattering microscopy, and most importantly, these signals are detected at high energies compared to the excitation wavelength. Therefore, the fluorescence background of the samples will not restrict the detection of the signals.

\subsection{Applications of SRS}

SRS are often used for imaging intracellular proteins and nucleic acids or monitoring glucose metabolism. Glucose is very important for cell anabolism and catabolic metabolism. It can provide the basis for a large amount of cell synthesis, produce ATP, and regulate intracellular redox potential. In addition, glucose metabolism plays an important role in the functioning of the body under any conditions, rightly. For example, one of the hallmarks of cancer is reprogrammed glucose metabolism with enhanced aerobic glycolysis. SRS imaging makes it possible for DNA condensation to be imaged with cell division and for these processes to be imaged in vivo [48]. With the development of technology, glucose metabolism has been tracked with positron emission tomography and magnetic resonance 
A

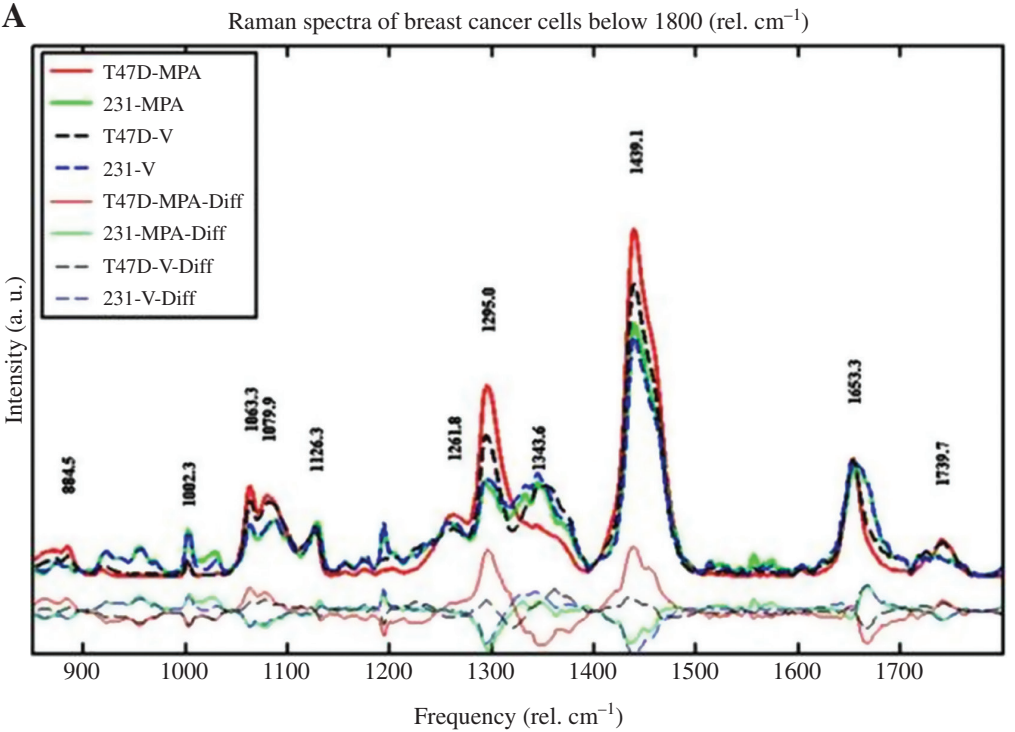

C

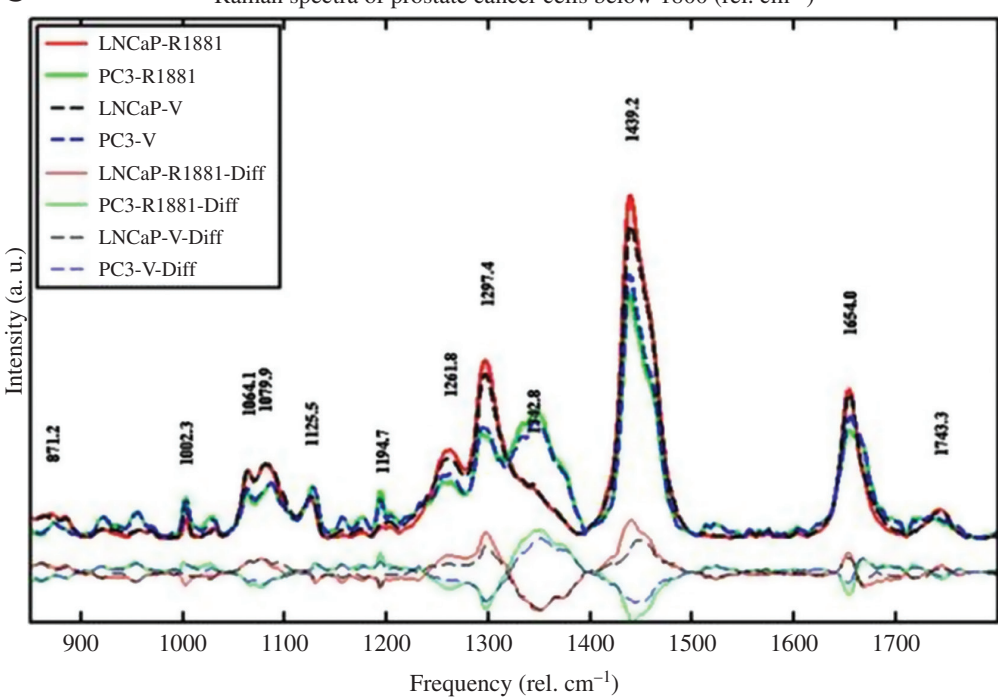

B Raman spectra of breast cancer cells above $2800\left(\right.$ rel. $\mathrm{cm}^{-1}$ )

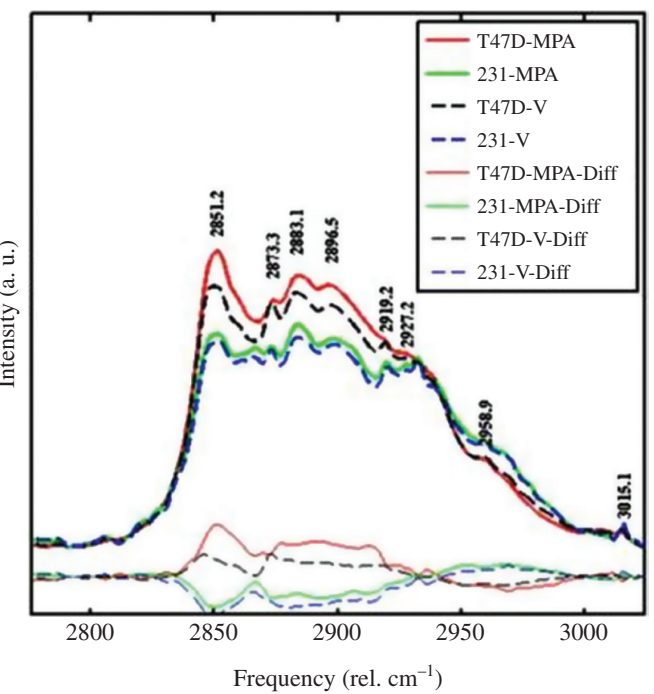

D Raman spectra of breast cancer cells above $2800\left(\right.$ rel. $\left.\mathrm{cm}^{-1}\right)$

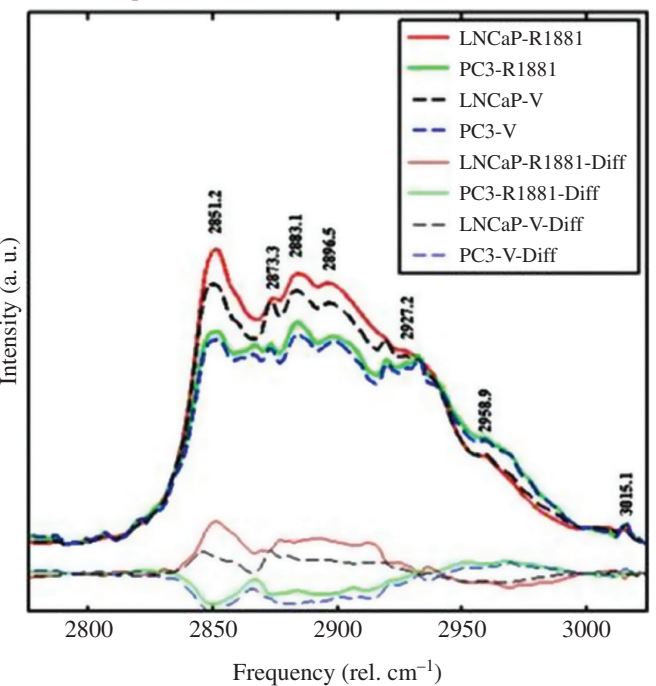

Figure 6: Mean Raman spectra of cancer cells in the hormone treatment group and the hormone receptor-deficient control group. Panels $(A)$ and $(B)$ are the low- and high-wavenumber regions, respectively, of spectra acquired from the breast cancer cell lines T47D and MDA-MB-231. Panels (C) and (D) are low and high wavenumber spectra, respectively, of prostate cancer cell lines LNCaP and PC3 [44].
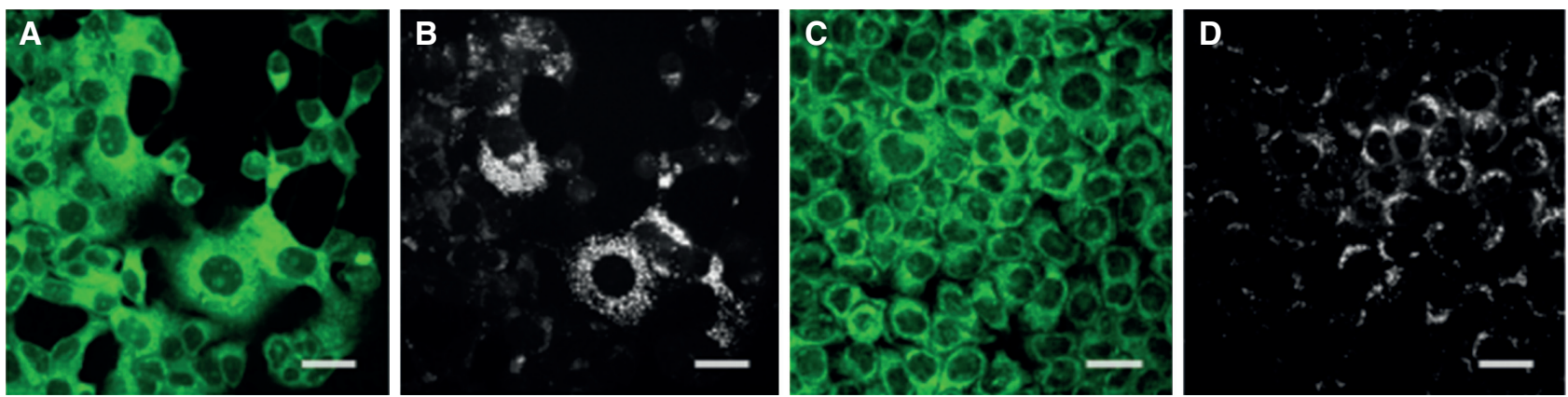

Figure 7: Autofluorescence and CARS images of treated versus vehicle control breast cancer cells.

(A) T47D-MPA autofluorescence image, (B) T47D-MPA CARS image, (C) T47D-V autofluorescence image, and (D) T47D-V CARS image [44]. 

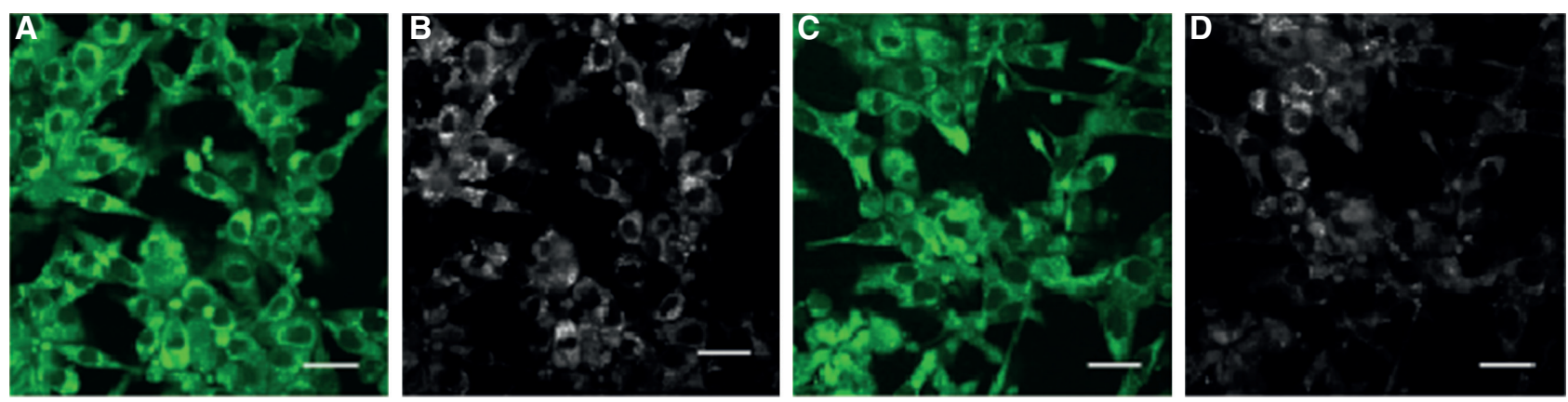

Figure 8: Autofluorescence and CARS images of treated versus vehicle control prostate cancer cells.

(A) LNCaP-R1881 autofluorescence image, (B) LNCaP-R1881 CARS image, (C) LNCaP-V autofluorescence image, and (D) LNCaP-V CARS image [44].

spectroscopy in clinical diagnosis, but both methods are limited by spatial resolution in intracellular studies. These techniques make use of a probe to detect the changes of glucose, so they provide only unfinished information about the glucose metabolism of cells. To provide a more complete picture of glucose metabolism, Long et al. used a two-color vibrational imaging method based on acetylene- and deuterium-labeled glucose analogs to observe glucose uptake and doping activity in the same living cell at the same time.

The above SRS imaging method can study glucose absorption and catabolism, and it has some advantages such as high resolution, specificity, and biocompatibility in mouse brain tissues. In particular, combining Ramanactive vibration probe with SRS microscopy provides a great method for bio-orthogonal chemical imaging of various small molecules in biological structures.

Using above technology, Long et al. observed the obvious activity of glucose metabolism in choroid plexus (CP) directly for the first time. In the unlabeled SRS image, lipids are represented in green and proteins in blue, and their distribution and combination patterns are shown (Figure 9A). The glucose combination channel and the glucose uptake channel both have obvious signals (Figure 9B). Therefore, two-color SRS imaging with subcellular resolution can be used for detecting synthesis and utilization of glucose in living brain tissues. They also show that bicolor ratio images can be used to quantify the glucose utilization and biomass contributions incorporated into the subcellular resolution of living systems.

\subsection{Applications of SHG}

Using in vivo imaging to determine the health of muscle cells may affect the diagnosis and observation of massive hereditary and developed muscle and heart diseases. In contrast, the technologies currently in use are limited due to inadequate resolution, absence of specificity, and invasiveness. There is a study that combined the intrinsic optical SHG of myosin with the new mathematical processing of striatum analysis to get a measure of muscle contractile integrity that is closely related to neuromuscular health. Plotnikov et al. used SHG microscopy to obtain digital images from skeletal muscle tissue, either normal or diseased. In healthy muscle fibers, the strips of sarcomatoid SHG are straight and regularly spaced, each almost perpendicular to the long axis of cell contraction. In contrast, in damaged cells, it shows a series of significant deviations from the standard, occasionally confined to subdomains within giant muscle fibers: excessive contraction or overexpansion of striated muscle spacing and incorrect positioning, tilt, or distortion of the belt. The strength of SHG is weakened or completely lost [49], as shown in Figure 10. Protein conformation and molecular order also can be probed by SHG microscopy. SHG is a nonlinear contrast source that, in some tissues, comes directly from an ordered array of unlabeled proteins. This property of SHG provides an exclusive chance to combine the advantages of NOMs with the detection of different levels of structural order in living tissues, from protein molecular conformations to supramolecular arrangements [50]. A study has developed a method for imaging sarcomere-level $\mathrm{Ca}^{2+}$ contractile coupling in cardiomyocytes in healthy and disease models. This method provides a model for studying the integrity of various cell models using SHG-2PF microscopy [51].

\subsection{Applications of TPEF}

Compared to single-photon microscopy, two-photon microscopy has the advantages of reducing background fluorescence of living cells and tissues, decreasing light damage and bleaching of biological samples, providing better 3D spatial positioning, and improving penetration depth. Small-molecule 2PF probes have been widely used in biological systems. Direct TP imaging of $\mathrm{H}_{2} \mathrm{~S}$ was successfully achieved in HeLa cells [52] (Figure 11). This 
A

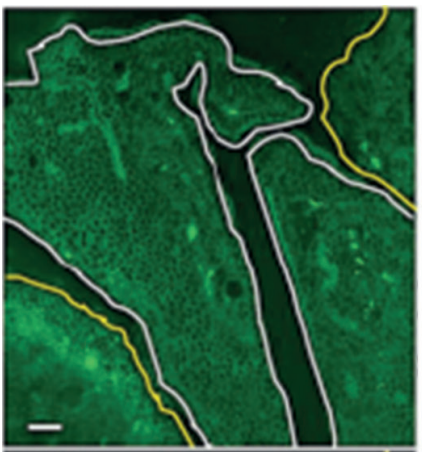

B
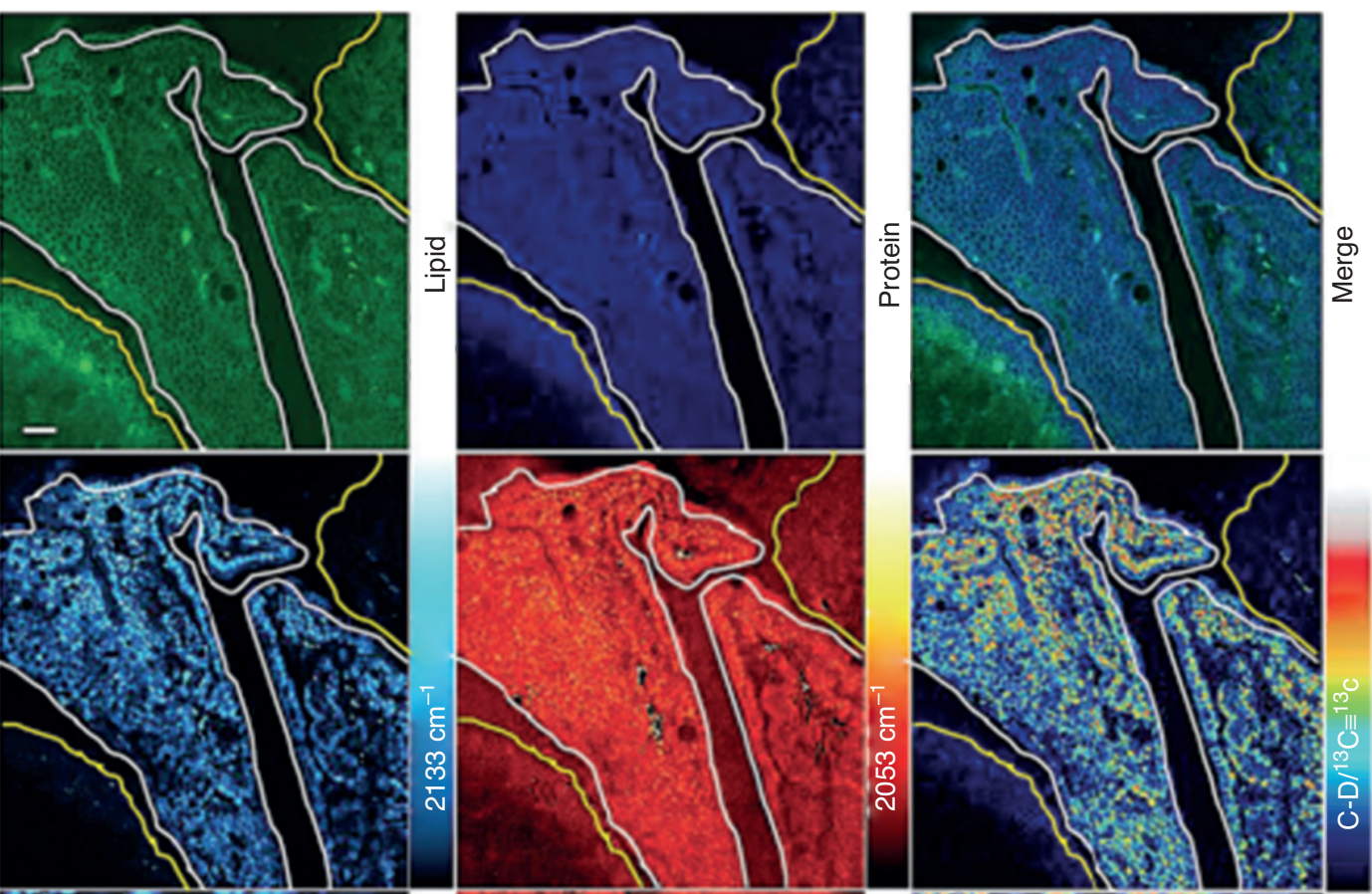

C
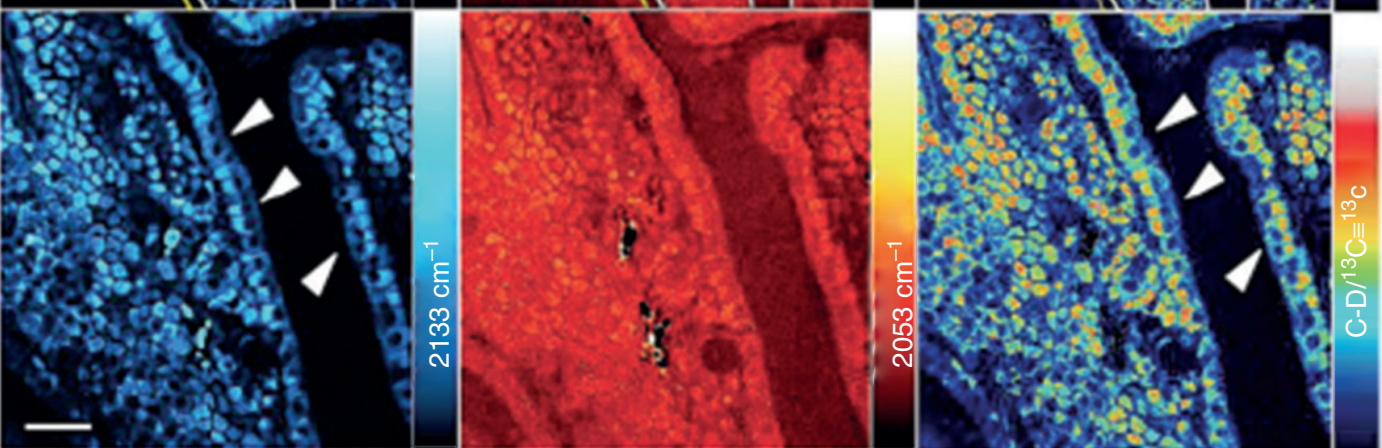

Figure 9: Imaging of $\mathrm{CP}$ with two-color glucose metabolism in ex vivo live mouse brain tissues.

(A) SRS images of lipid, protein and merged one, (B) SRS images of glucose incorporation and glucose uptake, and ratiometric image. CP shown as the white-solid line region and the nearby brain tissue shown as the yellow-solid line region, (C) SRS images of $2 \times$ zoomed in of the CP region [24].
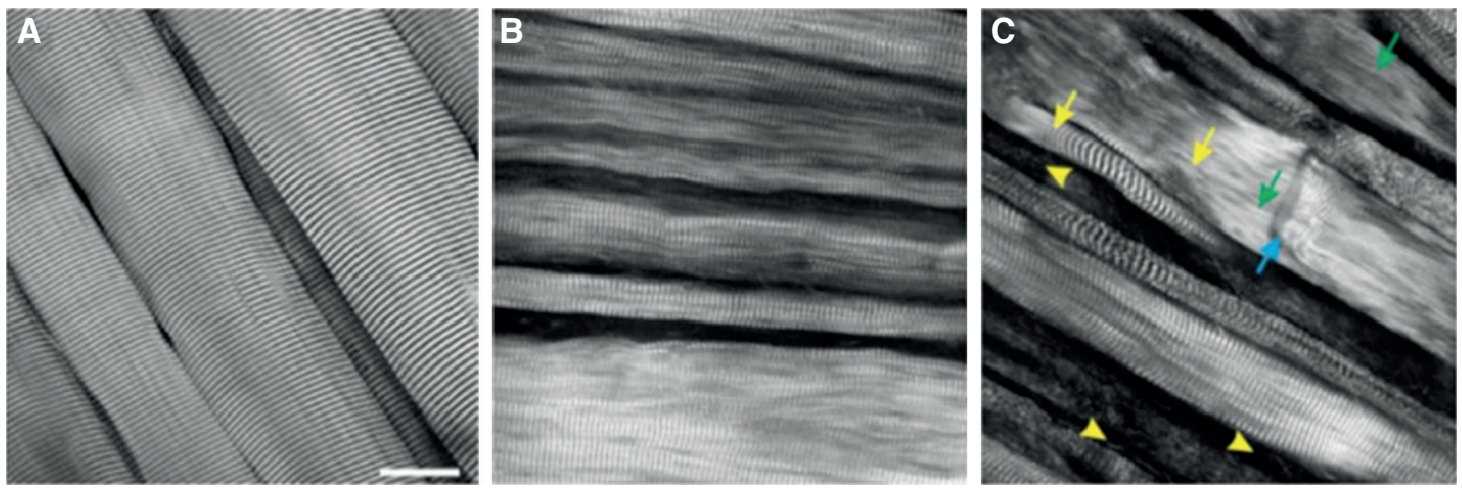

Figure 10: SHG imaging of normal and diseased muscle.

(A) Normal muscle, (B) and (C) diseased muscle. Yellow arrowheads mark gaps between myofibers; yellow and blue arrows label splits and ruptures, respectively, within the contractile lattice of myofibers; green arrows show myofiber areas with bright unstriated SHG [49]. 
shows that TP can be used for direct monitoring of $\mathrm{H}_{2} \mathrm{~S}$ in complex biological samples, showing its practical application value in biological systems.

\subsection{Applications of multiple methods}

Using CARS, SHG, and TPEF, Li et al. observed in what way collagen and bone co-compete to grow during the evolution of new organisms in vivo. Only the bone is completed compared to collagen, and the collagen starts to disappear gradually. Some of the nonlinear interactions between CARS, TPEF, and SHG take place only in very particular inherent biomolecules [53]. They allow for imaging without the need for exogenous stains, which are very convenient for in vivo applications. CARS can clearly image the bone growth of snails, and SHG can image the evolution of collagen and then observe the changes of their structure and morphology intuitively and visibly. Collagen is a kind of the most important proteins in living things. It is the mechanical macromolecule of the extracellular matrix. This allows imaging without the necessity to use dye materials, which can obtain easy and accurate imaging results in living organisms. CARS spectroscopy imaging is also used to study bones. TPEF can be used to study the metabolism of elastic fibers and certain cells. The specific process is shown in Figure 12.

\section{Applications of NOM for 2D materials}

Li et al. provided a high-resolution invasive NOM that could be used for the optical characterization of graphene. With these NOMs, the surface morphology of 2D materials can be clearly observed and their mass can be well characterized. The application of CARS technology in 2D material characterization has the unique advantage of being able to observe wrinkles and streaks that reflect the properties of graphene that grow on unpolished copper membranes, and the stretched samples showed clear graphene characteristics. The morphology and quality of graphene can be clearly observed in Figure 13. The image shows not only the individual imaging method but also the combination of several methods, and the relationship between them can be clearly seen [54].

Porous carbon materials are characterized by CARS microscopy. The slice of 2D CARS image is obtained by the $z$ scanning technique. On this basis, the 2D image of CARS scanned by $z$ is used to reconstruct the 3D image. The visualization results of the 3D rotation of CARS are given. Rotating 2D and 3D CARS images, we can easily obtain the surface and internal structure and properties of porous carbon materials, and the inverse symmetry breakage of porous carbon materials can be demonstrated by SHG imaging. TPEF displays that the porous carbon material has weak self-fluorescence characteristics (Figure 14). The study results show that NOMs are good optical analysis methods for porous carbon materials and have the potential of optical characterization of materials at microscale and nanoscale [23].

\section{Principle of plasmon-enhanced NOM}

NOMs is generally divided into two groups: coherent or incoherent. CARS, SRS, and SHG methods are coherent, and the phase of the generated optical signal is strictly defined by the incident light and the spatial structure of the target molecule. Some of the interaction signals of CARS, SHG, and TPEF occur simply in specific inherent biomolecules and 2D materials. The advantage is
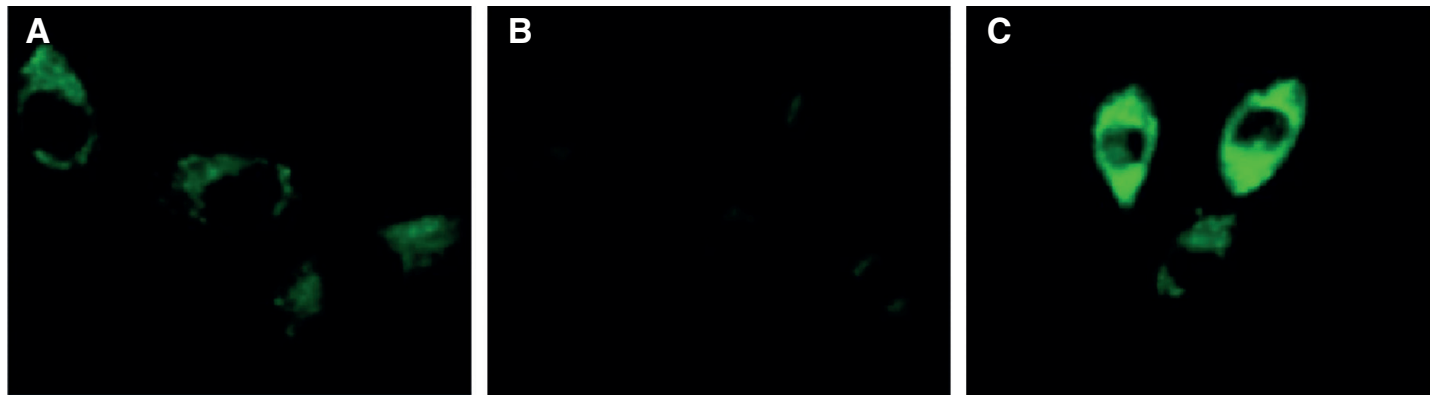

Figure 11: TP confocal microscopy images of $\mathrm{H}_{2} \mathrm{~S}$ detection in live HeLa cells.

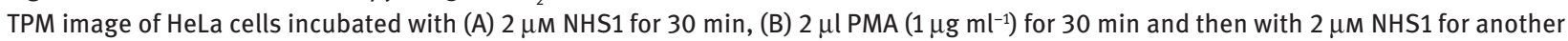
$30 \mathrm{~min}$, and (C) $2 \mu \mathrm{M}$ NHS1 for $30 \mathrm{~min}$ and then with $200 \mu \mathrm{M} \mathrm{NaSH}$ for another $30 \mathrm{~min}$ [52]. 

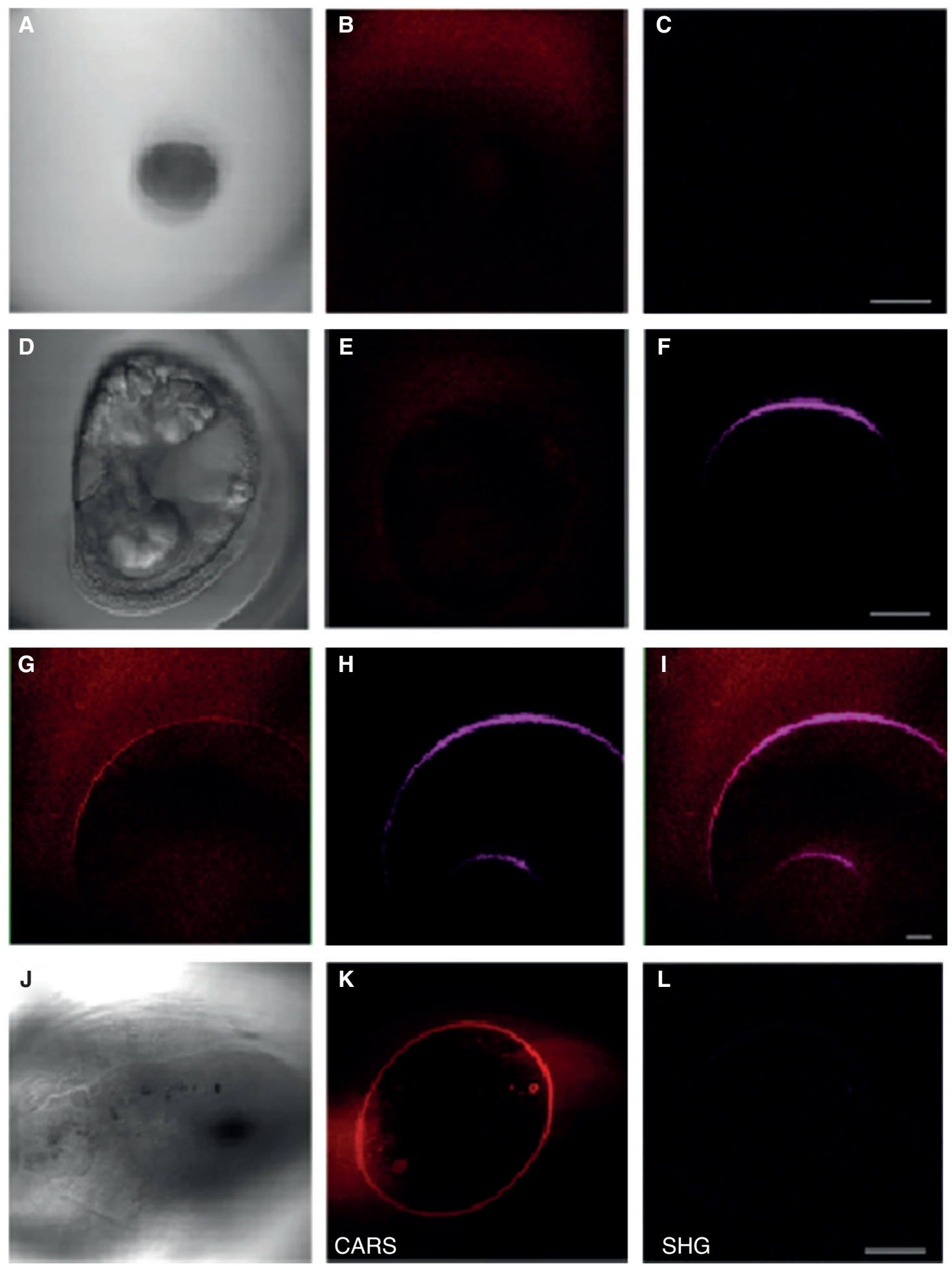

Figure 12: First 9 days of snail bone and collagen co-evolution process.

(A) The snail in bright field, (B) CARS image of snail bone ions of $\mathrm{CO}_{3}^{2-}$ and $\mathrm{PO}_{3}^{2-}$, (C) SHG image of snail's collagen on the first day, (D) The snail in bright field, (E) CARS image of snail bone ions of $\mathrm{CO}_{3}^{2-}$ and $\mathrm{PO}_{3}^{2-}$, (F) SHG image of snail's collagen on the third day, (G) CARS image of snail bone, (H) SHG image of snail's collagen, (I) The merged image of CARS and SHG on the fifth day, (J) The snail in bright field, (K) CARS image of the snail bone, (L) SHG image of the snail's collagen on the ninth day. The sample at the same day, there is the same scale bar. The scale bar is $100 \mu \mathrm{m}$ [53]. 

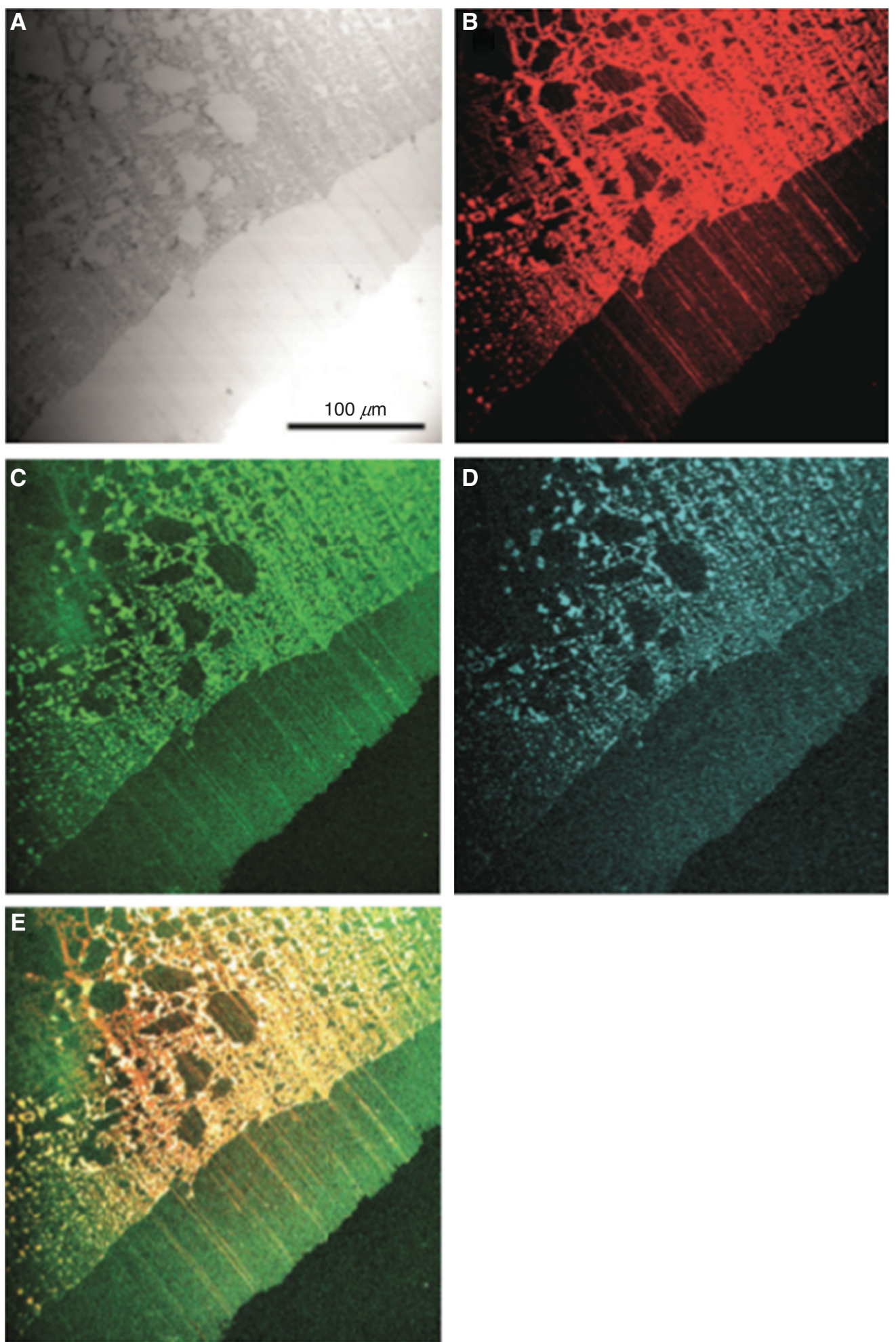

Figure 13: Images of multilayer graphene.

(A) Bright-field image of graphene, (B) CARS image of graphene, (C) TPEF image of graphene, (D) SHG image of graphene, and (E) merged image of CARS + TPEF+ SHG at Raman mode $1560 \mathrm{~cm}^{-1}$ [54].

that they can provide imaging and spectroscopy without the necessity for exogenous staining, so it is very convenient for optical characterization in vivo applications.
Incoherent microscopes such as TPEF produce optical signals with arbitrary phases. The optical nonlinearity is caused by the material properties of the interacting 

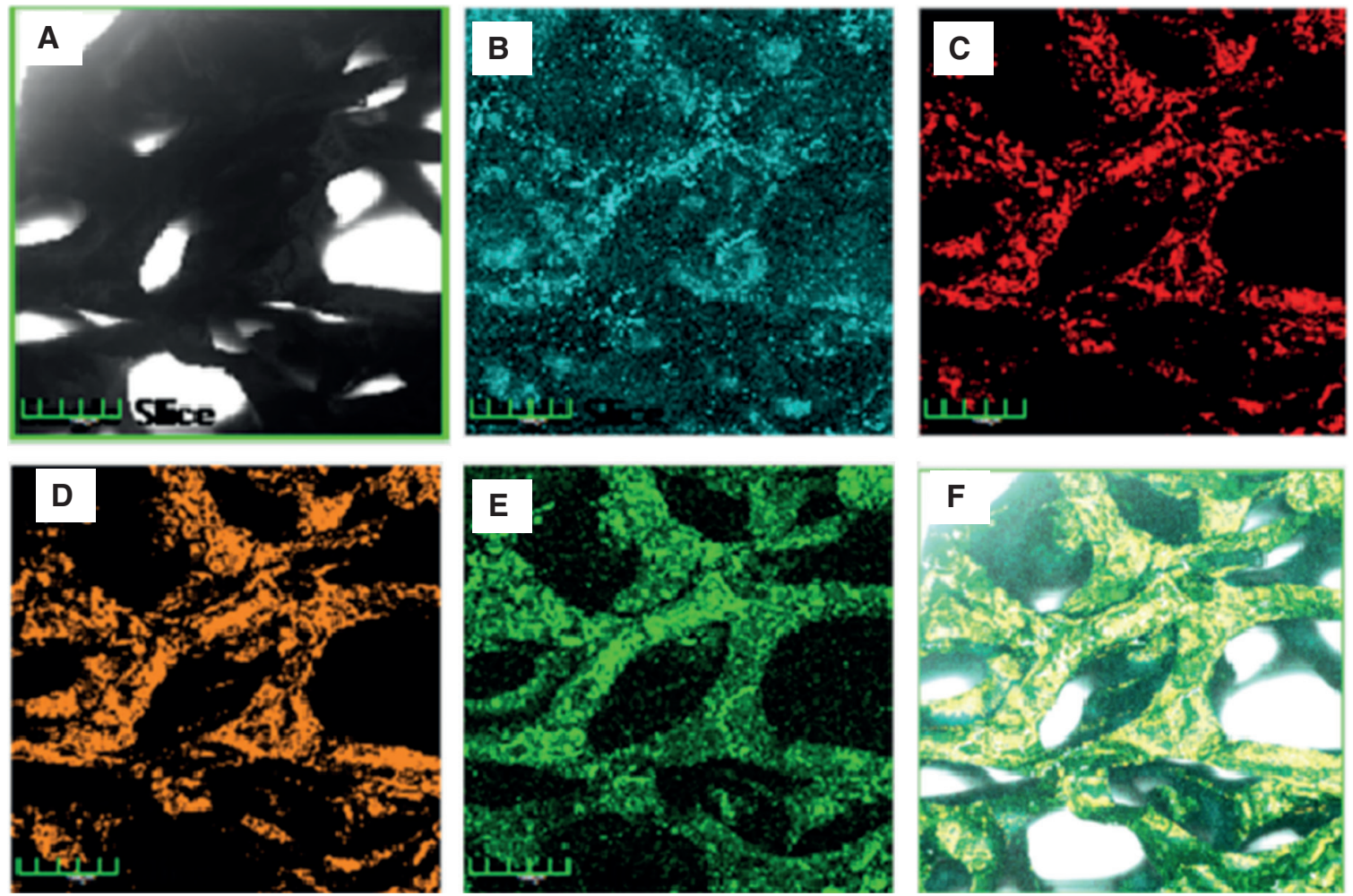

Figure 14: The images of nonlinear optical microscopy.

(A) Bright-field image of thinner layered porous carbon materials, (B) SHG, (C and D) CARS at 1587 and $1360 \mathrm{~cm}^{-1}$, respectively, (E) TPEF, and (F) merged bright-field image of SHG, CARS, and TPEF [23].

medium, and its inherent weakness is especially true for CARS. Because the coherent Raman signal is proportional to $\mathrm{N}(\mathrm{N}-1)$, where $\mathrm{N}$ is the number of molecules, the consequence of coherent enhancement of a single molecule decreases when $\mathrm{N}$ decreases and disappears with the decrease of $\mathrm{N}=1$. The measurement of coherent Raman imaging faces great challenges when the $\mathrm{N}$ is little, that is to say, the number of molecules is fewer when the difficulty is larger [55].

Plasmon can significantly enhance both incoherent and coherent nonlinear processes, including CARS [56], SHG [57, 58], hyper-Raman scattering [59], and TPEF [60]. Using plasma nanostructures to limit the light field at the nanoscale provides an effective approach for enhanced spectral signal

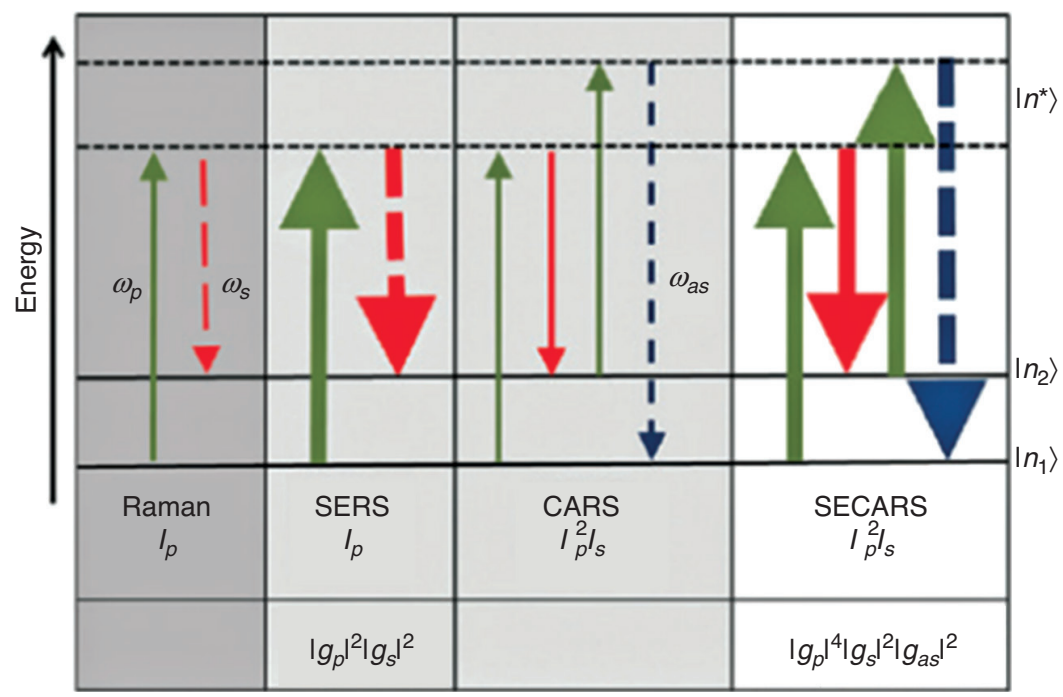

Figure 15: Energy diagrams of SECARS [61]. 
and optical imaging. Plasmas are caused by the cooperative coherent oscillations of photoexcited free electrons at optical frequencies. CARS can be obviously enhanced by the synthesis of Au@Ag nanorods. Surface-enhanced FAST CARS is also one kind of surface-enhanced CARS (SECARS) schemes. The energy diagrams are shown in Figure 15.

There is also a nonlinear optical method called surface-enhanced hyper-Raman scattering (SEHRS), and there are two incident photons whose frequency are $\omega_{0}$ into an outgoing photon frequency $\omega$ Raman shift compared to the second harmonic frequency $\left(\omega=2 \omega_{0}-\Delta \omega_{0}\right)$. In bulk solutions, the hyper-Raman strength is usually weak, making it difficult to measure the spectrum. Therefore, the enhancement factor of SEHRS is not common.

For the third-order nonlinear interaction, three incident photons interact with a solid dipole at the same time, annihilating and generating a new photon, and the frequency of new photo is related to incident photon frequency. If the frequencies of all incident photons are equal, if the energies of three incident photons are added together to emit a photon of three times the frequency, the process is called third harmonic generation (THG). The first implementation of SHG enhancement in surface plasmon resonances (SPRs) dates back to the 1970s. On rough silver surfaces, we found surface-enhanced SHG first [58]. Recently, Shen et al. created a 3D mushroom array model that significantly increased the scattering strength of plasmon-enhanced SHG [62]. It was found that the importance of localization SPR is considered to be a potential mechanism for metal nanoparticles (NPs) to enhance the weak SHG of biomolecules [63], which led to its widespread application in biological imaging.

In 1996, Kano and Kawata demonstrated for the first time that plasma-enhanced two-photon fluorescence and
SPR enhanced the full internal reflection two-photon fluorescence by 90 times. As learned earlier, plasma-enhanced two-photon self-fluorescence can be applied to imaging cells. Combining SPR field enhancement with nonlinear light emission can get clearer biological imaging photos successfully. One team that used this technology had imaged green fluorescent protein in the membrane of living monkey kidney fibroblasts [64].

\section{Applications of plasmon- enhanced NOMs in biology}

Although it has been more than 30 years since the first demonstration, few biological applications of SECARS have been discussed. Immunomicroscopy is the first application of immunostaining in biological imaging. White light (left) and immunofragments (right) are labeled by prostate biopsy as p63-antibody conjugation with plasma NPs, as shown in Figure 16 [65]. A type of biological application, the SECARS signal comes from the marked molecules that bind to plasma NPs and interact with prostate tissue through NPs-bound antibodies. Immunomicroscopy uses specific target nanoprobe staining that can be achieved simultaneously with a variety of dyes in a variety of spectral patterns. This makes it possible to image diverse cellular goals simultaneously. Various tags can realize great advantages in particular biological processes. The application of unlabeled SECARS in biological imaging is performed under a wide-area SECARS microscope with lipid structures that are placed on a 30-nm-thick plate gold substrate. A legible SECARS image of cholesterol oleate
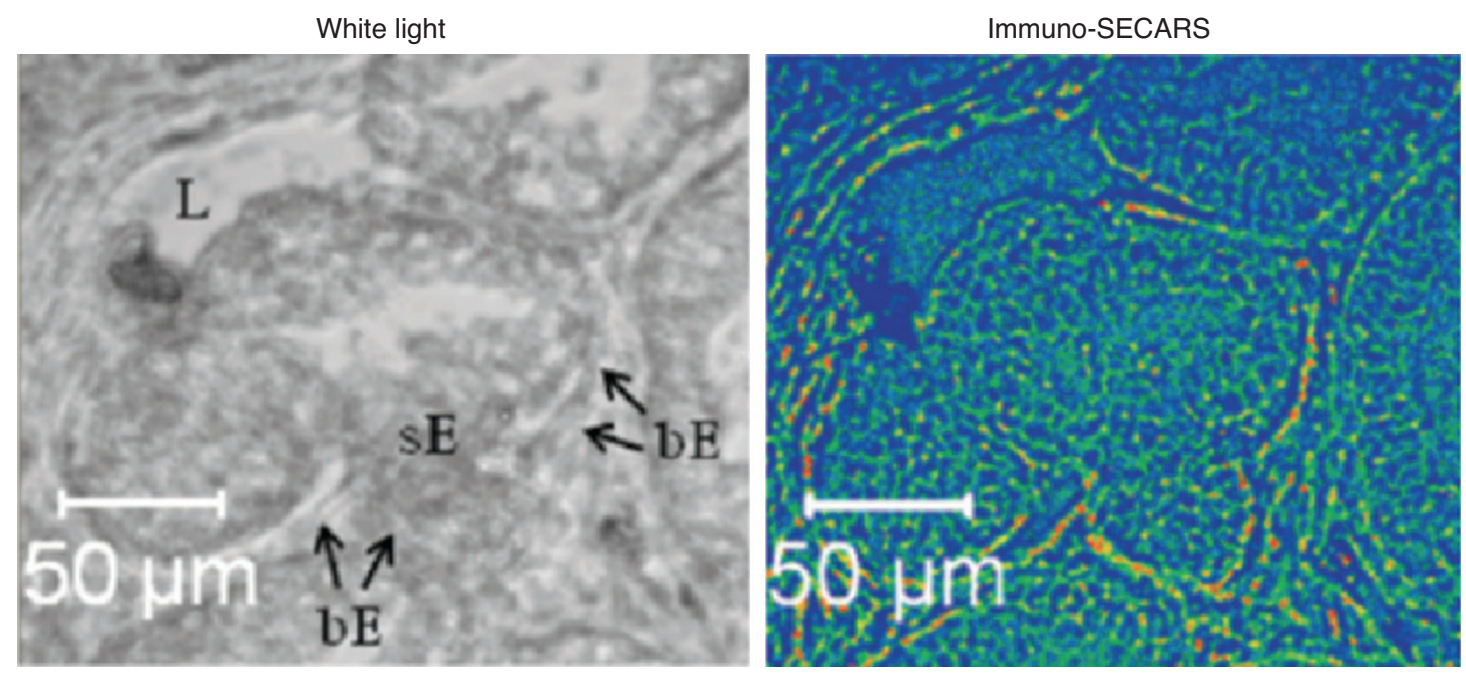

Figure 16: White light (left) and immune-SECARS (right) images of prostate tissue [64]. 

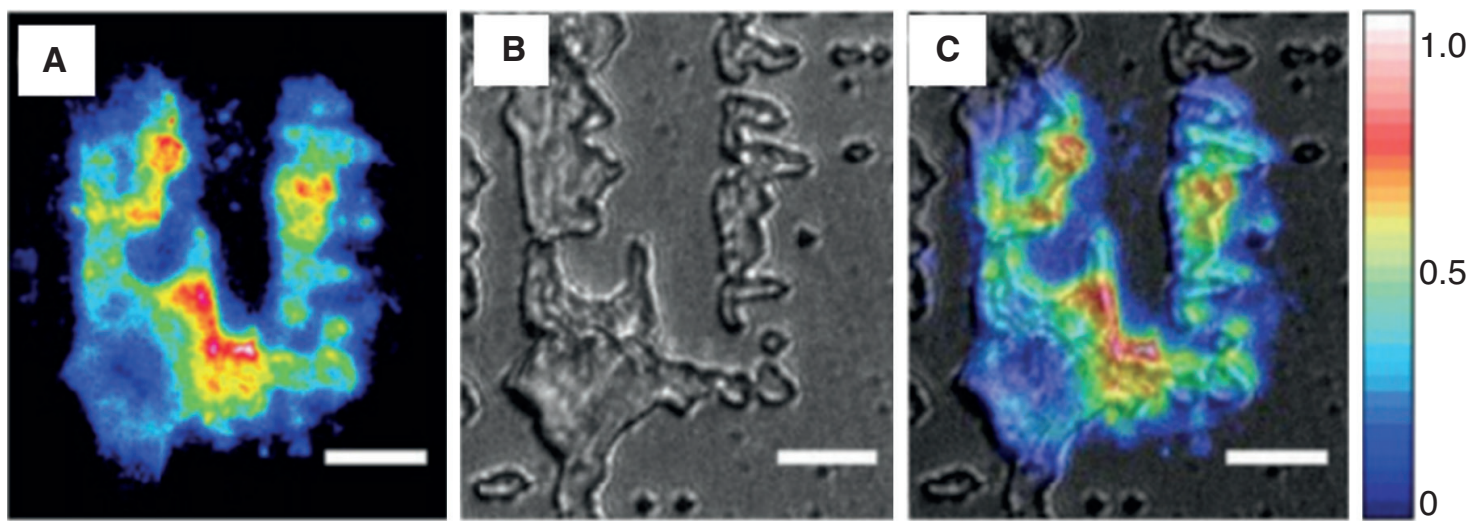

Figure 17: Wide-field SPP-based SECARS microscopy of lipid structures.

(A) SECARS, (B) transmission, and (C) overlapped SECARS and transmission images of a thick aggregate of cholesteryl oleate [66].

structure was gained by lighting densities, which is lower than conventional CARS with four orders of magnitude [66] (Figure 17). In addition to SHG, surface-enhanced THG was later discovered. Yelin et al. discussed the enhancement of the THG signal [67] (Figure 18).

\section{Applications of plasmon- enhanced NOMs for 2D materials}

In a paper, Mi et al. proved multiple SPR (MSPR) using $\mathrm{Au} @ \mathrm{Ag}$ nanorods at paired and fundamental frequencies of 400 and $800 \mathrm{~nm}$, respectively. Studies revealed that MSPR could considerably enhance NOM about twophoton CARS and TPEF and that Au@Ag nanorods could also meaningfully improve the two-photon CARS signals about 2D materials. Figure 19 shows the plasmon-enhanced TPEF image of Au@Ag nanorod covered surface of $\mathrm{g}-\mathrm{C}_{3} \mathrm{~N}_{4}$. Figure $19 \mathrm{C}$ is the TPEF of $\mathrm{g}-\mathrm{C}_{3} \mathrm{~N}_{4}$, and Figure 19D is the plasmon-enhanced TPEF of $\mathrm{g}-\mathrm{C}_{3} \mathrm{~N}_{4}$ covered with Au@Ag nanorod. Comparing Figure 19C and $\mathrm{D}$, it can be seen that TPEF is indeed enhanced by MSPR. The clarity of the latter is significantly higher than the former, proving that the Au@Ag nanorod-covered $\mathrm{g}-\mathrm{C}_{3} \mathrm{~N}_{4}$ could significantly enhance the TPEF signal.
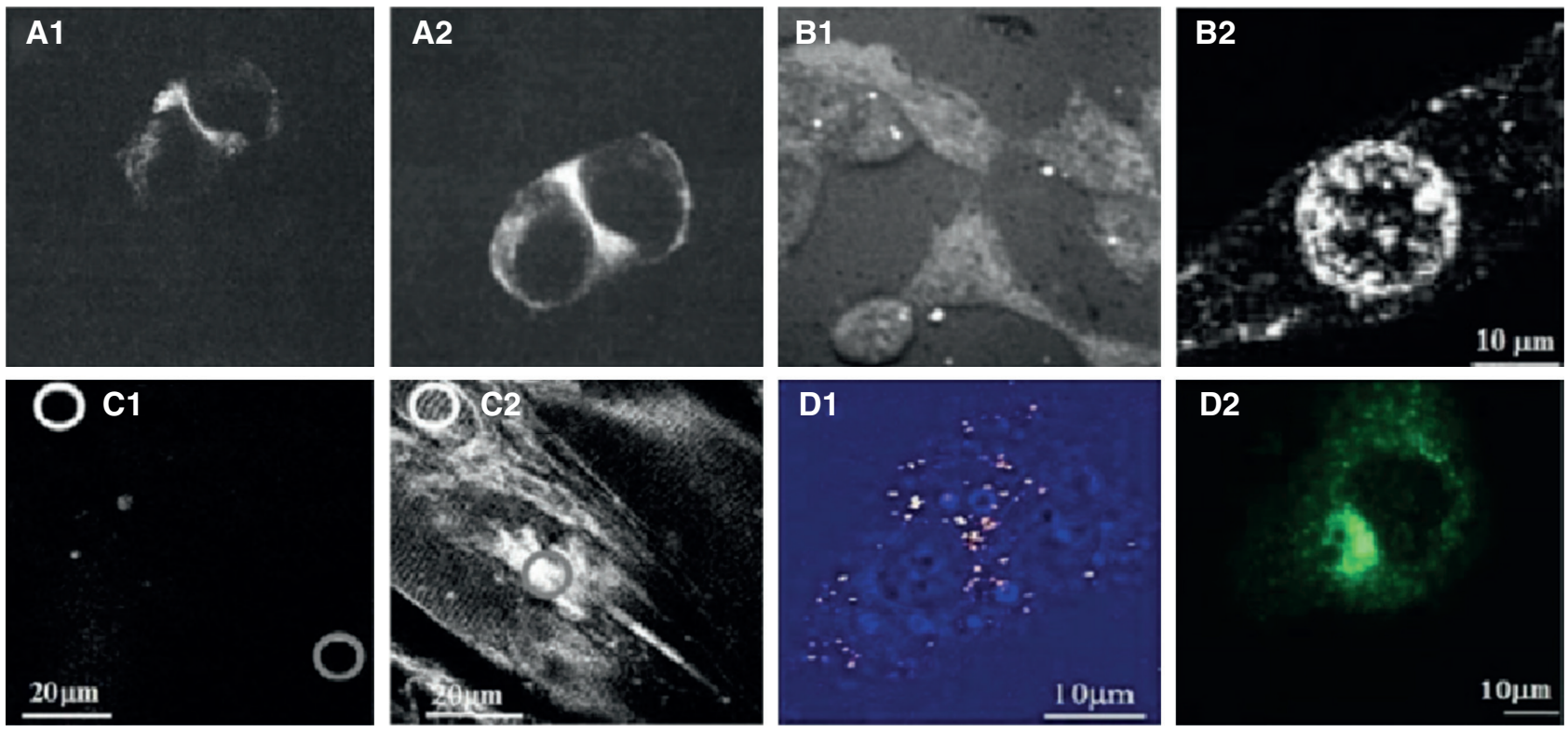

Figure 18: Plasmon-enhanced nonlinear biological imaging.

(A) SHG imaging of neuroblastoma cells: (A1) without NP and (A2) enhanced SHG signal after the addition of gold NP. (B1) Silver NP-enhanced THG image of wild-type MBT2 cells and (B2) plasmon-enhanced THG image of an NIH3T3 cell nucleus. (C1) Without and (C2) with silver NP-enhanced detection. (D1) Gold NP surface plasmon-enhanced autofluorescence image of live Chinese hamster ovary cells and (D2) surface plasmonenhanced two-photon total internal reflection fluorescence microscopy image of cultured monkey kidney fibroblast cell membrane [67]. 

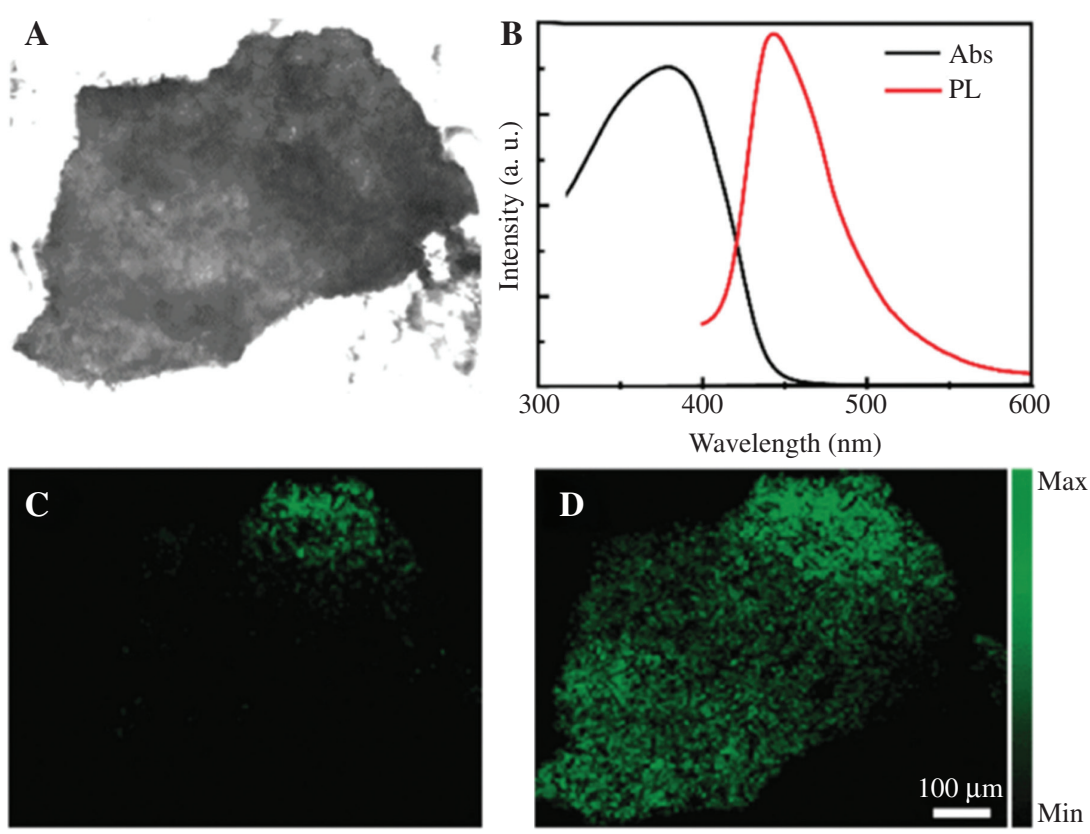

Figure 19: Optical characterization of $\mathrm{g}-\mathrm{C}_{3} \mathrm{~N}_{4}$.

(A) Bright-field image of $\mathrm{g}-\mathrm{C}_{3} \mathrm{~N}_{4}$, (B) absorption and PL spectra of $\mathrm{g}-\mathrm{C}_{3} \mathrm{~N}_{4}$, (C) TPEF of g- $\mathrm{C}_{3} \mathrm{~N}_{4}$, and (D) plasmon-enhanced TPEF of g-C $\mathrm{N}_{4}$ by MSPR [55].

They also measured the defect in the g- $\mathrm{C}_{3} \mathrm{~N}_{4}$ plasmareinforced CARS. Figure 20A is the bright-field image of defect $\mathrm{g}_{-} \mathrm{C}_{3} \mathrm{~N}_{4}$. It can be seen from the TPEF image that the photoluminescence (PL) strength is significantly reduced (Figure 20B). Even the CARS image is too weak to be detected (Figure 20C). Due to the obvious nonlinear electric field enhancement, the CARS signal can be greatly enhanced as shown in Figure 20G [55].
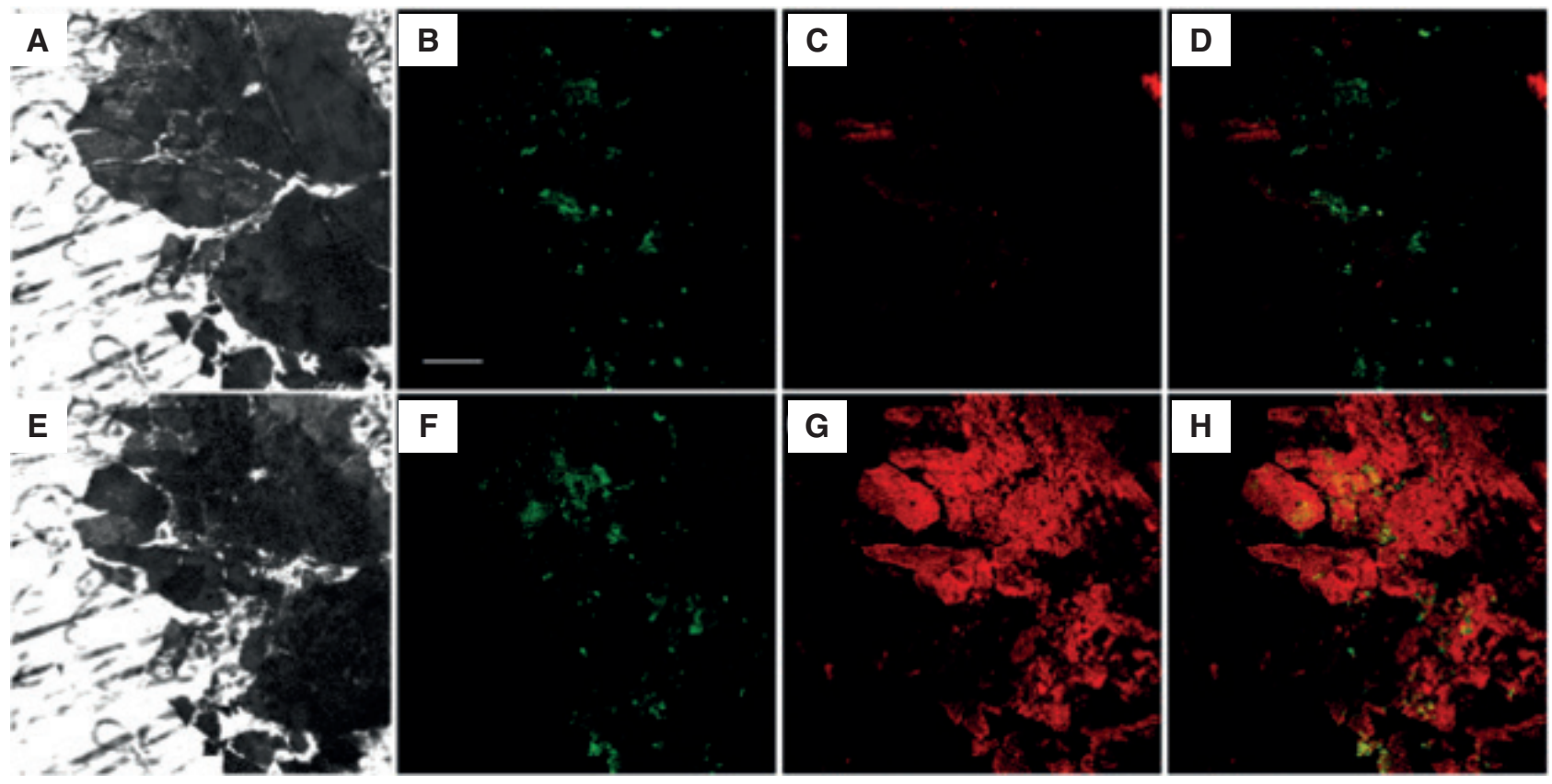

Figure 20: Optical characterization of plasmon-enhanced g- $\mathrm{C}_{3} \mathrm{~N}_{4}$.

(A) Bright-field image of g- $\mathrm{C}_{3} \mathrm{~N}_{4}$, (B) TPEF, (C) CARS, and (D) merged TPEF-CARS images and (E) bright-field image of defected g- $\mathrm{C}_{3} \mathrm{~N}_{4}$, (F) TPEF, (G) CARS, and (H) merged TPEF-CARS images [55]. 


\section{Conclusions}

We introduce the principles and applications of several NOMs. All of these NOMs have very broad applications in biology. CARS is an ideal tool for studying various samples; it greatly enhances the imaging speed through coherent amplification. In recent research progress, SRS has replaced the microscope CARS in many aspects. Without complex nonresonant background and phase limitations, SRS can observe and quantify samples more directly. In general, the main advantage of SHG over the more common TPEF is its ability to visualize sample assembly directly and extract more structural information using polarization and directional resolution without relying on extrapolation using exogenous labels. Multiple patterns can be combined to detect desired properties. Plasmon can significantly enhance the nonlinear optical process. Especially in the application of 2D materials, detailed internal information about materials can be easily obtained. As the prosperous nanophotonic technique of NOMs, the synthesis of probes and applications of plasmon-enhanced NOMs should be further investigated in detail.

Acknowledgments: This work was supported by the NSFC-BRICS STI Framework Program (grant 51861145309), the National Natural Science Foundation of China (grants 91436102, Funder Id: http://dx.doi. org/10.13039/501100001809, 11374353, and 11874084), and the Fundamental Research Funds for the Central Universities.

\section{References}

[1] Michiel M, Zumbusch A. Coherent anti-Stokes Raman scattering microscopy. ChemPhysChem 2007;8:2156-70.

[2] Cheng JX, Xie XS. Coherent anti-Stokes Raman scattering microscopy: instrumentation, theory, and applications. J Phys Chem B 2004;108:827-40.

[3] Cheng JX, Volkmer A, Xie XS. Theoretical and experimental characterization of coherent anti-Stokes Raman scattering microscopy. J Opt Soc Am B 2002;19:1363-75.

[4] Li R, Wang XX, Zhou Y, Zong H, Chen MD, Sun MT. Advances in nonlinear optical microscopy for biophotonics. J Nanophoton 2018;12:033007.

[5] Hong W, Karanja CW, Abutaleb NS, et al. Antibiotic susceptibility determination within one cell cycle at single bacterium level by stimulated Raman metabolic imaging. Anal. Chem 2018;90:3737-43.

[6] Kast RE, Tucker SC, Killian K, Trexler M, Honn KV, Auner GW. Emerging technology: applications of Raman spectroscopy for prostate cancer. Cancer Metastasis Rev 2014;33:673-93.
[7] Bradley J, Pope I, Masia F, et al. Quantitative imaging of lipids in live mouse oocytes and early embryos using CARS microscopy. Development 2016;143:2238-47.

[8] Ayyachamy S, Manivannan VS. Distance measures for medical image retrieval. Int J Imag Syst Tech 2013;23:9-21.

[9] Campagnola PJ, Wei MD, Lewis A, Loew LM. High-resolution nonlinear optical imaging of live cells by second harmonic generation. Biophys J 1999;77:3341-9.

[10] Hofemeier AD, Hachmeister H, Pilger C, et al. Label-free nonlinear optical microscopy detects early markers for osteogenic differentiation of human stem cells. Sci Rep 2016;6:26716.

[11] Min W, Freudiger CW, Lu S, Xie XS. Coherent nonlinear optical imaging: beyond fluorescence microscopy. Annu Rev Phys Chem 2011;62:507-30.

[12] Maker PD, Terhune RW. Study of optical effects due to an induced polarization third order in the electric field strength. Phys Rev 1965;137:801-18.

[13] Ploetz E, Laimgruber S, Berner S, Zinth W, Gilch P. Femtosecond stimulated Raman microscopy. Appl Phys B 2007;87:389-93.

[14] Freudiger CW, Min W, Saar BG, Lu S, Holtom GR, He C. Labelfree biomedical imaging with high sensitivity by stimulated Raman scattering microscopy. Science 2008;322:1857-61.

[15] Nandakumar P, Kovalev A, Volkmer A. Vibrational imaging based on stimulated Raman scattering microscopy. N J Phys 2009;11:033026-35.

[16] Macias-Romero C, Didier ME, Zubkovs V, et al. Probing rotational and translational diffusion of nanodoublers in living cells on microsecond time scales. Nano Lett 2014;14:2552-7.

[17] Theer P, Hasan MT, Denk W. Two-photon imaging to a depth of 1000 microns in living brains by use of a Ti: $\mathrm{Al}_{2} \mathrm{O}_{3}$ regenerative amplifier. Opt Lett 2003;28:1022-4.

[18] Liu ZR, Tang YH, Xu A, Lin WY. A new fluorescent probe with a large turn-on signal for imaging nitroreductase in tumor cells and tissues by two-photon microscopy. Biosens Bioelectron 2017;89:853-8.

[19] Boyd RW. Chapter 1: The nonlinear optical susceptibility. In: Nonlinear optics, 3rd ed. Academic Press, Burlington, 2008:1-67.

[20] Cheng JX, Jia YK, Zheng G, Xie XS. Laser-scanning coherent anti-Stokes Raman scattering microscopy and applications to cell biology. Biophys J 2002;83:502-9.

[21] Schie IW, Weeks T, Mcnerney GP, et al. Simultaneous forward and epi-CARS microscopy with a single detector by time-correlated single photon counting. Opt Express 2008;16:2168-75.

[22] Evans CL, Potma EO, Puoris'haag M, Cote D, Lin CP, Xie XS. Chemical imaging of tissue in vivo with video-rate coherent anti-Stokes Raman scattering microscopy. Proc Natl Acad Sci 2005;102:16807-12.

[23] Li R, Wang L, Mu X], Sun MT. Nonlinear optical characterization of porous carbon materials by CARS, SHG and TPEF. Spectrochim Acta A Mol Biomol Spectrosc 2019;214:58-66.

[24] Long R, Zhang L, Shi L, et al. Two-color vibrational imaging of glucose metabolism using stimulated Raman scattering. Chem Commun 2017;54:152-5.

[25] Ji M, Orringer DA, Freudiger CW, Ramkissoon S, Liu XH, Xie XS. Rapid, label-free detection of brain tumors with stimulated Raman scattering microscopy. Sci Transl Med 2013;5:201ra119.

[26] Saar BG, Freudiger CW, Reichman J, Stanley CM, Holtom GR, Xie XS. Video-rate molecular imaging in vivo with stimulated Raman scattering. Science 2010;330:1368-70. 
[27] Freudiger CW, Min W, Saar BG, et al. Label-free biomedical imaging with high sensitivity by stimulated Raman scattering microscopy. Science 2008;322:1857-61.

[28] Ito T, Obara Y, Misawa K. Single-beam phase-modulated stimulated Raman scattering microscopy with spectrally focused detection. J Opt Soc Am B 2017;34:1004.

[29] Lee HJ, Cheng JX. Imaging chemistry inside living cells by stimulated Raman scattering microscopy. Methods 2017;128:119-28.

[30] Freudiger CW, Min W, Holtom GR, Xu B, Dantus M, Xie XS. Highly specific label-free molecular imaging with spectrally tailored excitation-stimulated Raman scattering (STE-SRS) microscopy. Nat Photonics 2011;5:103-9.

[31] Conklin MW, Eickhoff JC, Riching KM, et al. Aligned collagen is a prognostic signature for survival in human breast carcinoma. Am J Pathol 2011;178:1221-32.

[32] Kirkpatrick ND, Brewer MA, Utzinger U. Endogenous optical biomarkers of ovarian cancer evaluated with multiphoton microscopy. Cancer Epidemiol Biomarkers Prev 2007;16:2048-57.

[33] Lacomb R, Nadiarnykh O, Campagnola PJ. Quantitative SHG imaging of the diseased state osteogenesis imperfecta: experiment and simulation. Biophys J 2008;94:4504-14.

[34] Schenke-Layland K, Xie J, Angelis E, et al. Increased degradation of extracellular matrix structures of lacrimal glands implicated in the pathogenesis of Sjogren's syndrome. Matrix Biol 2008;27:53-66.

[35] Strupler M, Pena AM, Hernest M, et al. Second harmonic imaging and scoring of collagen in fibrotic tissues. Opt Express 2007;15:4054-65.

[36] Kwon GP, Schroeder JL, Amar MJ, Remaley AT, Balaban RS. Contribution of macromolecular structure to the retention of low-density lipoprotein at arterial branch points. Circulation 2008;117:2919-27.

[37] Lin SJ, Wu R, Tan HY, et al. Evaluating cutaneous photoaging by use of multiphoton fluorescence and second-harmonic generation microscopy. Opt Lett 2005;30:2275-7.

[38] Lo W, Teng SW, Tan HY, et al. Intact corneal stroma visualization of GFP mouse revealed by multiphoton imaging. Microsc Res Technol 2006;69:973-5.

[39] Han M, Giese G, Bille JF. Second harmonic generation imaging of collagen fibrils in cornea and sclera. Opt Express 2005;13:5791-7.

[40] Huang JX, Li N, Wang QQ, Gu YQ, Wang P. A lysosome-targetable and two-photon fluorescent probe for imaging endogenous $\beta$-galactosidase in living ovarian cancer cells. Sens Actuators B 2017;246:833-9.

[41] Natter K, Kohlwein SD. Yeast and cancer cells - common principles in lipid metabolism. Biochim Biophys Acta 2013;1831:314-26.

[42] Baade PD, Youlden DR, Krnjacki LJ. International epidemiology of prostate cancer: geographical distribution and secular trends. Mol Nutr Food Res 2009;53:171-84.

[43] Stanford JL, Wicklund KG, McKnight B, Daling JR, Brawer MK. Vasectomy and risk of prostate cancer. Cancer Epidemiol Biomarkers Prev 1999;8:881-6.

[44] Potcoava MC, Futia GL, Aughenbaugh J, Schlaepfer IR, Gibson EA. Raman and coherent anti-Stokes Raman scattering microscopy studies of changes in lipid content and composition in hormone-treated breast and prostate cancer cells. J Biomed Opt 2014;19:111605.
[45] Schlaepfer IR, Hitza CA, Gijónb MA, Bergmana BC, Eckela RH, Jacobsena BM. Progestin modulates the lipid profile and sensitivity of breast cancer cells to docetaxel. Mol Cell Endocrinol 2012;363:111-21.

[46] Lui H, Zhao J, McLean D, Zeng H. Real-time Raman spectroscopy for in vivo skin cancer diagnosis. Cancer Res 2012;72:2491-500.

[47] Surmacki J, Musial J, Kordek R, Abramczyk H. Raman imaging at biological interfaces: applications in breast cancer diagnosis. Mol Cancer 2013;12:1-12.

[48] Zhang X, Roeffaers MB, Basu S, et al. Label-free live-cell imaging of nucleic acids using stimulated Raman scattering microscopy. ChemPhysChem 2012;13:1054-9.

[49] Plotnikov SV, Kenny AM, Walsh SJ, et al. Measurement of muscle disease by quantitative second-harmonic generation imaging. J Biomed Opt 2008;13:044018.

[50] Vanzi F, Sacconi L, Cicchi R, Pavone FS. Protein conformation and molecular order probed by second-harmonic-generation microscopy. J Biomed Opt 2012;17:060901.

[51] Awasthi S, Izu LT, Mao Z, et al. Multimodal SHG-2PF imaging of microdomain $\mathrm{Ca}^{2+}$-contraction coupling in live cardiac myocytes. Circ Res 2016;118:e19-28.

[52] Mao GJ, Wei TT, Wang XX, et al. High-sensitivity naphthalenebased two-photon fluorescent probe suitable for direct bioimaging of $\mathrm{H}_{2} \mathrm{~S}$ in living cells. Anal Chem 2013;85:7875-81.

[53] Li R, Wang L, Mu XJ, Chen MD, Sun MT. Biological nascent evolution of snail bone and collagen revealed by nonlinear optical microscopy. J Biophotonics 2019;12:e201900119.

[54] Li R, Zhang YJ, Xu XF, Zhou Y, Chen MD, Sun MT. Optical characterizations of two-dimensional materials using nonlinear optical microscopies of CARS, TPEF, and SHG. Nanophotonics 2018;7:873-81.

[55] Mi XH, Wang YY, Li R, Sun MT, Zhang ZL, Zheng HR. Multiple surface plasmon resonances enhanced nonlinear optical microscopy. Nanophotonics 2019;8:487-93.

[56] Ichimura T, Hayazawa N, Hashimoto M, Inouye Y, Kawata S. Tip-enhanced coherent anti-Stokes Raman scattering for vibrational nanoimaging. Phys Rev Lett 2004;92:220801-4.

[57] Campagnola PJ, Clark HA, Mohler WA, Lewis A, Loew LM. Second-harmonic imaging microscopy of living cells. J Biomed Opt 2001;6:277-86.

[58] Chen CK, Castro ARB, Shen YR. Surface-enhanced secondharmonic generation. Phys Rev Lett 1981;46:145-8.

[59] Golab J, Sprague J, Carron K, Schatz G, Van Duyne R. A surface enhanced hyper-Raman scattering study of pyridine adsorbed onto silver: experiment and theory. J Chem Phys 1988;88:7942-51.

[60] Sanchez EJ, Novotny L, Xie XS. Near-field fluorescence microscopy based on two-photon excitation with metal tip. Phys Rev Lett 1999;82:4014-7.

[61] Steuwe C, Kaminski CF, Baumberg JJ, Mahajan S. Surfaceenhanced coherent anti-Stokes Raman scattering on nanostructured gold surfaces. Nano Lett 2011;11:5339-43.

[62] Shen SX, Shan JJ, Shih T-M, et al. Competitive effects of surface plasmon resonances and interband transitions on plasmonenhanced second-harmonic generation at near-ultraviolet frequencies. Phys Rev Appl 2020;13:024045.

[63] Chen B, Estrada LC, Hellriegel C, Gratton E. Nanometer-scale optical imaging of collagen fibers using gold nanoparticles. Biomed Opt Express 2011;2:511-9. 
[64] He RY, Su YD, Cho KC, et al. Surface plasmon-enhanced twophoton fluorescence microscopy for live cell membrane imaging. Opt Express 2009;17:5987-97.

[65] Schlucker S, Salehi M, Bergner G, et al. Immuno-surfaceenhanced coherent anti-Stokes Raman scattering microscopy: immune-histochemistry with target-specific metallic nanoprobes and nonlinear Raman microscopy. Anal Chem 2011;83:7081-5.
[66] Fast A, Kenison JP, Syme CD, Potma EO. Surface-enhanced coherent anti-Stokes Raman imaging of lipids. Appl Opt 2016;55:5994-6000.

[67] Yelin D, Oron D, Thiberge S, Moses E, Silberberg Y. Multiphoton plasmon-resonance microscopy. Opt Express 2003;11:1385-91. 